TRANSACTIONS OF THE

AMERICAN MATHEMATICAL SOCIETY

Volume 356, Number 3, Pages 853-880

S 0002-9947(03)03157-X

Article electronically published on October 21, 2003

\title{
ON THE WEYL TENSOR OF A SELF-DUAL COMPLEX 4-MANIFOLD
}

\author{
FLORIN ALEXANDRU BELGUN
}

\begin{abstract}
We study complex 4-manifolds with holomorphic self-dual conformal structures, and we obtain an interpretation of the Weyl tensor of such a manifold as the projective curvature of a field of cones on the ambitwistor space. In particular, its vanishing is implied by the existence of some compact, simply-connected, null-geodesics. We also show that the projective structure of the $\beta$-surfaces of a self-dual manifold is flat. All these results are illustrated in detail in the case of the complexification of $\mathbb{C P}^{2}$.
\end{abstract}

\section{INTRODUCTION}

Twistor theory, created by Penrose [16], establishes a close relationship between conformal Riemannian geometry in dimension 4, and (almost) complex geometry in dimension 3. In particular, to a Riemannian manifold $M$ for which the part $W^{-}$of the Weyl tensor vanishes identically (self-dual), one associates its twistor space $Z$, a complex 3-manifold containing rational curves with normal bundle $\mathcal{O}(1) \oplus \mathcal{O}(1)$ (called twistor lines), and admitting a real structure with no fixed points [1], [6], [3].

The space of such curves is a complex 4-manifold $\mathbf{M}$ [10] with a holomorphic conformal structure and is, therefore, a conformal complexification of $M$ [1], [6], [3].

As the conformal geometry of $M$ is encoded by the complex geometry of $Z$, we ask ourselves what holomorphic object on $Z$ corresponds to $W^{+}$, the Weyl tensor of the self-dual manifold $M$. It seems that this question, although natural, has not been considered in the literature, and maybe a reason for that is that the answer appears to be a highly non-linear object.

This object is more easily understood in the framework of complex-Riemannian geometry (see Section 2): For a self-dual (complex) 4-manifold $\mathbf{M}$, its (local) twistor space is then defined as the 3 -manifold of $\beta$-surfaces (some totally geodesic isotropic surfaces; see Section 21). Following LeBrun [14, we further introduce the (locallydefined) space $B$ of complex null-geodesics of $\mathbf{M}$ (ambitwistor space).

The ambitwistor space $B$ and (in the self-dual case) the twistor space $Z$ completely describe the conformal structure of $\mathbf{M}$. In particular, a null-geodesic $\gamma$ in $\mathbf{M}$ corresponds to the set of twistor lines in $Z$ tangent to a 2-plane [13. The union of these curves, called the integral $\alpha$-cone of $\gamma$ (see Section B), is lifted to a (linearized) $\alpha$-cone in $T_{\gamma} B$. Our first result (Theorem 1) is that the Weyl tensor of $\mathbf{M}$ is equivalent to the projective curvature (see Section 4) of the field of $\alpha$-cones

Received by the editors February 27, 2000.

2000 Mathematics Subject Classification. Primary 53C21, 53A30, 32Qxx. 
on $B$. In particular, if such a cone is flat, then $W^{+}$vanishes on certain isotropic planes in $\mathbf{M}$.

We use Theorem 1 to investigate global properties of a self-dual manifold $\mathbf{M}$ : If the integral $\alpha$-cone of $\gamma$ is part of a smooth surface in $Z$, then the linearized $\alpha$-cone is flat (Theorems 2, $2^{\prime}$ ). In particular, the space $\mathbf{M}_{0}$ of rational curves of $Z$ with normal bundle $\mathcal{O}(1) \oplus \mathcal{O}(1)$ is compact iff $Z \simeq \mathbb{C P}^{3}$. On the other hand, it is known, from a theorem of Campana 4, that, for a compact twistor space $Z, \mathbf{M}_{0}$ can be compactified within the space of analytic cycles iff $Z$ is Moishezon. It appears then that the conformal structure does not extend smoothly to the compactification.

A good illustration of what happens in the non-flat (self-dual) case is the KählerEinstein manifold $\mathbb{C P}^{2}$ whose twistor space is known to be the manifold of flags in $\mathbb{C}^{3}$ [1; see Section 7 .

A first application of Theorem 2 is that a civilized (a topological assumption on the conformal manifold, permitting the global construction of its (ambi-)twistor space - see [12], [15], and Section 2) self-dual manifold containing a compact nullgeodesic is conformally flat, and the null-geodesic is simply-connected (Theorem 3). If we assume, in addition, that the compact null-geodesic is simply-connected, then the above result can be deduced, using Theorem $2^{\prime}$, for any self-dual 4-manifold, and also (using the LeBrun correspondence [12]) for the case of a conformal complex 3-manifold [2]; In fact, we have recently proven 2 that the existence of a compact, simply-connected, null-geodesic on an $n$-dimensional conformal complex manifold implies its conformal flatness, for any $n \geq 3$ (different methods are used for $n>3$ ).

Another application of Theorem 2 is that the family of twistor lines on a twistor space never induces a projective structure on it, unless the twistor space is an open set in $\mathbb{C P}^{3}$ (Corollary 1).

The isotropic, totally geodesic surfaces (called $\beta$-surfaces) in a self-dual manifold $\mathbf{M}$ have a projective structure, given by the null-geodesics of $\mathbf{M}$ contained in it (Section 6). We show that it is flat (i.e. locally equivalent to $\mathbb{C P}^{2}$ ) (Corollary 2), and we obtain a classification of the compact $\beta$-surfaces of a self-dual 4-manifold (Theorem 4).

The paper is organized as follows. In Section 2 we recall the classical results of the twistor theory (especially for complex 4-manifolds), in Section 3 we introduce the $\alpha$-cones on the (ambi-)twistor space, and, in Section 4 we prove the equivalence between the projective curvature of the latter and the Weyl tensor $W^{+}$of $\mathbf{M}$. Section 15 is devoted to the proof of some results of the type "compactness implies conformal (projective) flatness": Theorems 2, 2' and 3 mentioned above. We study the projective structure of $\beta$-surfaces in Section 6 , and we illustrate the above results on the special case of the self-dual manifold $\mathbb{C P}^{2}$ in Section 7 .

\section{Preliminaries}

The content of this paper makes use of complex-Riemannian geometry, which is obtained by analogy from Riemannian geometry by replacing the field $\mathbb{R}$ by $\mathbb{C}$ (e.g. a complex metric is a non-degenerate symmetric complex-bilinear form on the tangent space), and all classical results hold, naturally with the exception of those making use of partitions of unity. We will often omit the prefix complex- when referring to geometric objects, and we will always consider them, unless otherwise stated, in the framework of complex-Riemannian geometry. 
2.1. Conformal complex 4-manifolds. Let $\mathbf{M}$ be a 4-dimensional complex manifold. A conformal structure is defined, as in the real case [5], by an everywhere non-degenerate section $c$ of the complex bundle $S^{2}\left(T^{*} \mathbf{M}\right) \otimes L^{2}$, where $L$ is a given line bundle of scalars of weight 1 , and $L^{4} \simeq \kappa^{-1}$, the anti-canonical bundle of M. (While on an oriented real manifold such a line bundle always exists, being topologically trivial, in the complex case the existence of $L^{2}$, a square root of the anti-canonical bundle, is submitted to some topological restrictions.) From now on, only holomorphic conformal structures will be considered; thus $L$ is a holomorphic bundle and $c$ a holomorphic section of $S^{2}\left(T^{*} \mathbf{M}\right) \otimes L^{2}$. (In fact, all we need to define the conformal structure $c$ on the 4-manifold $\mathbf{M}$ is just the holomorphic bundle $L^{2}$; in odd dimensions the situation is different; see [2].)

As in the real case, $c$ is locally represented by symmetric bilinear forms on $T \mathbf{M}$, or local sections in $L^{2}$, but global representative metrics do not exist, in general.

For each point $x \in \mathbf{M}$, there is an isotropy cone $C_{x}$ in the tangent space $T_{x} \mathbf{M}$, which uniquely determines the conformal structure $c$. In the associated projective space, $\mathbb{P}\left(T_{x} \mathbf{M}\right) \simeq \mathbb{C P}^{3}$, the cone $C_{x}$ projects onto the non-degenerate quadratic surface $\mathbb{P}\left(C_{x}\right)$, which is actually a ruled surface isomorphic to $\mathbb{C P}^{1} \times \mathbb{C P}^{1}$. We thus get 2 families of complex projective lines contained in $\mathbb{P}(C)$, that is, 2 families of isotropic 2-planes in $C \subset T \mathbf{M}$, respectively called $\alpha$-planes and $\beta$-planes. This choice corresponds to the choice of an orientation of $\mathbf{M}$. On a real 4-manifold an orientation is chosen by picking a class of "positive" volume forms (which is not possible in this complex framework) or by choosing one of the two possible Hodge operators compatible with the conformal structure $*: \Lambda^{2} \mathbf{M} \rightarrow \Lambda^{2} \mathbf{M}$ (which can also be done in our complex case, [17]). As $*$ is a symmetric involution, $\Lambda^{2} \mathbf{M}$ decomposes into $\Lambda^{+} \mathbf{M} \oplus \Lambda^{-} \mathbf{M}$, consisting in \pm 1 -eigenvectors of $*$, respectively called self-dual and anti-self-dual 2-forms; the isotropic vectors in $\Lambda^{+} \mathbf{M}$ and $\Lambda^{-} \mathbf{M}$ are then exactly the decomposable elements $u \wedge v \in \Lambda^{ \pm} \mathbf{M}$, with $u, v \in \mathbf{M}$.

Definition 1. An $\alpha$-plane $F^{\alpha}$ (resp. a $\beta$-plane $F^{\beta}$ ) in $T \mathrm{M}$ is a 2-plane such that $\Lambda^{2} F^{\alpha}$ (resp. $\Lambda^{2} F^{\beta}$ ) is a self-dual (resp. anti-self-dual) isotropic line in $\Lambda^{2} \mathbf{M}$.

Remark. The $\alpha$ - and $\beta$-planes can be interpreted in terms of spinors. The structure group of the tangent bundle $T \mathbf{M}$ is restricted to the conformal orthogonal complex group, $C O(4, \mathbb{C}):=\left(O(4, \mathbb{C}) \times \mathbb{C}^{*}\right) /\{ \pm \mathbf{1}\}$, where $O(4, \mathbb{C}):=\{A \in$ $\left.G L(4, \mathbb{C}) \mid A^{t} A=\mathbf{1}\right\}$, by the given conformal structure of $\mathbf{M}$. The choice of an orientation is the further restriction of this group to the connected component of the identity, $C O_{0}(4, \mathbb{C}):=S O(4, \mathbb{C}) \times \mathbb{C}^{*}$, where $S O(4, \mathbb{C}):=O(4, \mathbb{C}) \cap S L(4, \mathbb{C})$. Consider a local metric $g$ in the conformal class $c$. We have then locally defined Spin structures, and associated Spin bundles $V_{+}, V_{-}$, as in the real case [1], [18. They are rank 2 complex vector bundles, and for each local section of $L^{2}$ (i.e. a metric in $c$ ), each of them is equipped with a (complex) symplectic structure $\omega_{+} \in \Lambda^{2} V_{+}, \omega_{-} \in \Lambda^{2} V_{-}$, respectively. Then we locally have $T \mathbf{M} \simeq V_{+} \otimes V_{-}$, and $g=\omega_{+} \otimes \omega_{-}$, for the fixed metric $g \in c . \alpha$ - (resp. $\beta$-) planes are then nothing but the isotropic 2-planes obtained by fixing the first (resp. the second) factor in the above tensor product:

Proposition 1 ([17]). An $\alpha$-plane, resp. $\beta$-plane $F \subset T_{x} \mathbf{M}$ is a complex plane $\psi_{+} \otimes V_{-}$, resp $V_{+} \otimes \psi_{-}$, where $\psi_{+} \in V_{+} \backslash\{0\}$, resp. $\psi_{-} \in V_{-} \backslash\{0\}$.

The $\alpha$-planes in $T_{x} \mathbf{M}$ are thus indexed by $\mathbb{P}\left(V_{+}\right)_{x}$, and $\beta$-planes by $\mathbb{P}\left(V_{-}\right)_{x}$, and these projective bundles are globally well-defined on $\mathbf{M}[1]$. 
Remark. It is obvious that a change of orientation interchanges the $\alpha$ - and $\beta$-planes; the same is true for self-duality and anti-self-duality, to be defined below.

For a local metric $g$ in $c$, we denote by $R^{g}$ its Riemannian curvature, and by $W$ the Weyl tensor, i.e. the trace-free component of $R^{g}$, which is known to be independent of the chosen metric within the conformal class [5]. It splits into two components $W^{+}, W^{-}$, and the easiest way to see that is the spinorial decomposition of the space of the curvature tensors $\mathcal{R} \subset \Lambda^{2} \otimes \Lambda^{2}$ ([1, [18], [19]), obtained from the relation $T \mathbf{M}=V_{+} \otimes V_{-}$and some of the Clebsch-Gordan identities [18. We have

$$
\mathcal{R}=\mathcal{S} \oplus \mathcal{B} \oplus \mathcal{W}^{+} \oplus \mathcal{W}^{-},
$$

where $\mathcal{S}$ is the complex line of scalar curvature tensors, ("diagonally") included in $\Lambda^{2} V_{+} \oplus \Lambda^{2} V_{-} \simeq \mathbb{C} \oplus \mathbb{C}, \mathcal{B}=S^{2} V_{+} \otimes S^{2} V_{-}$is the space of trace-free Ricci tensors, and $\mathcal{W}^{+}=S^{4} V_{+}, \mathcal{W}^{-}=S^{4} V_{-}$are the spaces of self-dual, resp. anti-self-dual Weyl tensors (where $S^{p} V_{ \pm}$denotes the $p$-symmetric power of $V_{ \pm}$).

The curvature $R^{g}$ restricted to any $\alpha$-plane $F$ yields a weighted bilinear symmetric form $R^{F}$ on $\Lambda^{2} F$, i.e. a section in $L^{2} \otimes\left(\Lambda^{2} F \otimes \Lambda^{2} F\right)^{*}$ :

$$
(g, X \wedge Y) \stackrel{R^{F}}{\longmapsto} g\left(R^{g}(X, Y) X, Y\right) .
$$

Proposition 2. The (weighted) bilinear form $R^{F}$ depends only on the self-dual Weyl tensor, and this one is completely determined by the (weighted) values of $R^{F}$ for all $\alpha$-planes $F$.

We have the same result for $\beta$-planes.

Proof. Let $F=\psi_{+} \otimes V_{-}$be an $\alpha$-plane, let $X=\psi_{+} \otimes \varphi_{1}, Y=\psi_{+} \otimes \varphi_{2} \in F$, and suppose, for simplicity, that $\omega_{-}\left(\varphi_{1}, \varphi_{2}\right)=1$, so $X \wedge Y \in \Lambda^{2} F$ is identified with the element $\psi_{+} \otimes \psi_{+} \in S^{2} V_{+}$. Then it is easy to see that $R^{F}$, evaluated on $X \wedge Y$, is nothing but the evaluation of $R \in S^{2}\left(\Lambda^{2} \mathbf{M}\right) \supset \mathcal{R}$ on $(X \wedge Y) \otimes(X \wedge Y) \simeq$ $\psi_{+} \otimes \psi_{+} \otimes \psi_{+} \otimes \psi_{+} \in S^{4} V_{+}$, which depends only on the positive (or self-dual) part of the Weyl tensor. To prove the second assertion, we remark that $W^{+}$, being a quadrilinear symmetric form on $V_{+}$, can be identified with a polynomial of degree 4 on $V_{+}$, which is determined by its values.

Definition 2. A conformal structure $c$ on a 4-manifold $\mathbf{M}$ is called self-dual (resp. anti-self-dual) iff $W^{-}=0$ (resp. $\left.W^{+}=0\right)$.

Remark. In general, geodesics on a conformal manifold depend on the chosen metric, with the exception of the isotropic ones (or null-geodesics). Therefore the existence of totally geodesic surfaces tangent to $\alpha$ - (resp. $\beta$-) planes is a property of the conformal structure alone.

\subsection{Twistor spaces.}

Definition 3. An $\alpha$-surface (resp. $\beta$-surface) $\alpha \subset \mathbf{M}$ is a maximal, totally geodesic surface in $\mathbf{M}$ whose tangent space at any point is an $\alpha$-plane (resp. $\beta$-plane).

On the other hand, any totally geodesic, isotropic surface in $\mathbf{M}$ is included in an $\alpha$ - or in a $\beta$-surface.

Definition 4 ([16], 17]). If, at any point $x \in \mathbf{M}$, and for any $\alpha$ - (resp. $\beta$-) plane $F \subset T_{x} \mathbf{M}$, there is an $\alpha$ - (resp. $\beta$-) surface tangent to $F$ at $x$, we say that the family of $\alpha$ - (resp. $\beta$-) planes is integrable. 
Theorem ([1], 17]). The family of $\alpha$ - (resp. $\beta$-) planes of a conformal 4-manifold $(\mathbf{M}, c)$ is integrable if and only if the conformal structure $c$ is anti-self-dual (resp. self-dual).

The integrability of $\alpha$-planes is equivalent to the integrability (in the sense of Frobenius) of a distribution $H^{\alpha}$ of 2-planes on the total space of the projective bundle $\mathbb{P}\left(V_{+}\right)$. Namely, let $g$ be a local metric in the conformal class $c$, and let $\nabla$ be its Levi-Civita connection. $\nabla$ induces a connection in the bundle $\mathbb{P}\left(V_{+}\right)$, thus a horizontal distribution $H$, isomorphic to $T \mathbf{M}$ via the bundle projection. Let $H^{\alpha}$ be the 2-dimensional subspace of $H_{F}$-where $F \in \mathbb{P}\left(V_{+}\right)$is an $\alpha$-plane in $T_{x} \mathbf{M}$ which projects onto $F \subset T_{x} \mathbf{M}$. It can easily be shown (as in [17, see also [1]) that the tautological 2-plane distribution $H^{\alpha}$ is independent of the metric $g$. Then $\alpha$-surfaces are canonically lifted as integrable manifolds of the distribution $H^{\alpha}$. For a geodesically convex open set of $\mathbf{M}$, one can prove (see [15]) that the space of these integrable leaves is a complex 3 -manifold. (This point of view is closely related to that of [1, about the integrability of the canonical almost complex structure of the real twistor space.)

The same remark can be made about $\beta$-surfaces.

Remark. The existence, for any point $x \in \mathbf{M}$, of an $\alpha$-surface containing $x$ does not imply, in general, the integrability of the whole family of $\alpha$-planes: in the conformal self-dual (but not anti-self-dual) manifold $\mathbf{M}=\mathbb{C P}^{2} \times\left(\mathbb{C P}^{2}\right)^{*} \backslash \mathcal{F}$ (the complexification of $\left.\mathbb{C P}^{2},[1]\right)$, the surfaces $\left(\{x\} \times\left(\mathbb{C P}^{2}\right)^{*}\right) \cap \mathbf{M}$ and $\left(\mathbb{C P}^{2} \times\{y\}\right) \cap \mathbf{M}$ are all $\alpha$-surfaces, see Section 7 .

Remark. In the real framework, the twistor space of a real Riemannian 4-manifold $M^{\mathbb{R}}$ is the total space $Z^{\mathbb{R}}$ of the $S^{2}$-bundle of almost-complex structures on $T M^{\mathbb{R}}$, compatible with the conformal structure and the (opposite) orientation; it admits a natural almost-complex structure $\mathcal{J}$, equal, at the point $J \in Z^{\mathbb{R}}$, to the complex structure of the fibers on the vertical space $T_{J}^{\vee} Z^{\mathbb{R}}$, and to $J$ itself on the horizontal space (induced by the Levi-Civita connection). Such a complex structure $J$ is equivalent to an isotropic complex 2-plane in $T M \otimes \mathbb{C}$, thus to an $\alpha$-or $\beta$-surface (depending on the conventions), which then becomes the space of vectors of type $(1,0)$ for $J$; as the integrability of the almost-complex structure $\mathcal{J}$ can be expressed as the Frobenius condition applied to $T^{(1,0)} Z^{\mathbb{R}}$, it is equivalent to the integrability of the family of $\alpha$-, resp. $\beta$-planes.

The Penrose construction associates to an (anti-)self-dual manifold $\mathbf{M}$ the space $Z$ of $\alpha$ - (resp. $\beta$-)surfaces of $\mathbf{M}$; we have seen above that $Z$ admits complex-analytic maps, but it may be non-Hausdorff. This is why we need to introduce the following condition; see also [15:

Definition 5. An (anti-)self-dual manifold $\mathbf{M}$ is called civilized iff the space $Z^{\alpha}$ (resp. $Z^{\beta}$ ) of integral leaves of the distribution $H^{\alpha}$ (resp. $H^{\beta}$ ) in $\mathbb{P}\left(V_{+}\right)$(resp. $\mathbb{P}\left(V_{-}\right)$) is a complex 3-manifold, and the projection $p^{+}: \mathbb{P}\left(V_{+}\right) \rightarrow Z^{\alpha}$ (resp. $p^{-}$: $\left.\mathbb{P}\left(V_{-}\right) \rightarrow Z^{\beta}\right)$ is a submersion.

In this case, the manifold $Z^{\alpha}$ (resp. $Z^{\beta}$ ) - which is the space of $\alpha$-surfaces (resp. $\beta$-surface) of $\mathbf{M}$ - is called the $\alpha$ - (resp. the $\beta$-)twistor space of $\mathbf{M}$.

From now on, we suppose that $(\mathbf{M}, c)$ is a self-dual complex analytic 4-manifold. As any point $x \in \mathbf{M}$ has a geodesically convex neighborhood $U$ [21] (which is, therefore, civilized [15]), we can construct $Z^{U}$, the $\beta$-twistor space (for short, twistor 
space) of $U$. As most results of this paper are infinitesimal, we will usually suppose (with no loss of generality) that $\mathbf{M}$ is civilized (for example, by replacing $\mathbf{M}$ by $U$ ).

We recall now the correspondence between differential geometric objects on $\mathbf{M}$ and complex analytic objects on its twistor space $Z$ ([1], [17]; see also [12, [13, [18]).

$\beta$-surfaces $\beta \subset \mathbf{M}$ correspond to points $\bar{\beta} \in Z$, by definition, and the set of $\beta$-surfaces passing through a point $x \in \mathbf{M}$ is a complex projective line $Z_{x}$, with normal bundle isomorphic (non-canonically) to $\mathcal{O}(1) \oplus \mathcal{O}(1)$ (where $\mathcal{O}(1)$ is the dual of the tautological bundle $\mathcal{O}(-1)$ on $\left.\mathbb{C P}^{1}\right)$ (1], [17]; see also [3]). Such a curve will be called a twistor line.

In fact, the family of twistor lines in $Z$ permits us to recover $\mathbf{M}$ and its conformal structure, at least locally, by the reverse Penrose construction: The normal bundle $N_{x}$ of a line $Z_{x}$ in $Z$ has the property $H^{1}\left(N_{x}, \mathcal{O}\right)=0$; thus, by a theorem of Kodaira [10], the space $\mathbf{M}_{0}$ of projective lines in $Z$ having the above normal bundle is a smooth complex manifold whose tangent space at a point $x \simeq Z_{x} \subset Z$ is canonically isomorphic to the space of global sections of the normal bundle $N_{x}$ of $Z_{x}$ (thus $\mathbf{M}_{0}$ has dimension 4 ). The conformal structure of $\mathbf{M}_{0}$ is described by its isotropy cone, which corresponds to the sections of $N_{x}$ having at least one zero (as such a section decomposes as 2 sections of $\mathcal{O}(1)$, the vanishing condition means that they both vanish at the same point, which is a quadratic condition on the sections of $N_{x}$ ). We thus get a conformal diffeomorphism from $\mathbf{M}$ to an open set of $\mathbf{M}_{0}$.

2.3. Ambitwistor spaces. We remark that $\mathbb{P}\left(V_{-}\right)$is an open set of the projective tangent bundle of $Z$, as $Z$ is the space of leaves of $\mathbb{P}\left(V^{-}\right)$, but it is important to note that, in general, the reverse inclusion is not true (i.e. not every direction in $Z$ is tangent to a line corresponding to a point in $\mathbf{M}$, or, equivalently, $\beta$-surfaces are not compact $\mathbb{C P}^{2}$ 's, in general, see Section 5 ).

For example, if $\mathbf{M}=\mathbb{C P}^{2} \times \mathbb{C P}^{*} \backslash \mathcal{F}$ (with the notation in Section 7), $\mathbb{P}\left(V_{-}\right)$is an open subset in the $\mathbb{C P}^{2}$-bundle $\mathbb{P}(T Z) \rightarrow Z$, consisting of the set of directions transverse to the contact structure of $Z$ (see Subsection 7.4). $\mathbb{P}\left(V_{-}\right)$is thus, in this case, a rank 2 affine bundle over $Z$.

Another canonical $\mathbb{C P}^{2}$-bundle on $Z$, that is, $\mathbb{P}\left(T^{*} Z\right) \rightarrow Z$, leads to the ambitwistor space $B$, which is by definition the space of null-geodesics of $\mathbf{M}$ [13]. It is an open set of the projective cotangent bundle of $Z$ (or, equivalently, the Grassmannian of 2-planes in $T Z$ ) [13]. More precisely, a plane $F \subset T_{\bar{\beta}} Z$ corresponds to a null-geodesic $\gamma \subset \mathbf{M}$ (contained in $\beta$ ) if it is tangent to at least one projective line $Z_{x}$, corresponding to a point $x \in \mathbf{M}$.

To see that, let $x$ be a point in $\mathbf{M}, \beta$ a $\beta$-surface passing through $x$, i.e. $\bar{\beta} \in Z$ and $Z_{x}$ contains $\bar{\beta}$; let $F \subset T_{\bar{\beta}} Z$ be a plane tangent to $Z_{x}$. As small deformations of $Z_{x}$ still correspond to points of $\mathbf{M}$, we consider the twistor lines which are tangent to $F$. They correspond to a (path-connected) set of points on a curve $\gamma \subset \beta$, which will turn out to be a null-geodesic. Indeed, all we have to prove is $\ddot{\gamma}=0(\bmod \dot{\gamma})$, and $\dot{\gamma}_{x}$ corresponds to a section $\eta$ of $N_{x}$, vanishing at $\bar{\beta} \in Z_{x}$; as $N_{x} \simeq \mathcal{O}(1) \oplus \mathcal{O}(1), \eta$ is determined by its derivative at $\bar{\beta}$, which is a linear map $T_{\bar{\beta}} \rightarrow F / T_{\bar{\beta}}$ (the infinitesimal deformation of the direction of $Z_{x}$ within $F$ ). As the points of $\gamma$ correspond to lines tangent to $F$, we have that $\ddot{\gamma}_{x}$ corresponds to a section of $N_{x}$ collinear to $\eta$; thus $\gamma$ satisfies the equation of a (non-parameterized) geodesic. See [12], [15], and Section 4 for details. 
Example. The space of null-geodesics of $\mathbf{M}=\mathbb{P}(E) \times \mathbb{P}(E)^{*} \backslash \mathcal{F}$ is the total space of a $\mathbb{C} \times \mathbb{C P}^{1}$-bundle over $Z=\mathcal{F}$, the flag manifold (see Section 7); a 2-plane $F \subset T_{(L, l)} \mathcal{F}$ which corresponds to a null-geodesic in $\mathbf{M}$ is identified either with a projective diffeomorphism $\varphi: \mathbb{P}(l) \rightarrow \mathbb{P}\left(L^{o}\right)$ (Subsection 7.4, case 3), or with a point $A \subset l, A \neq L$, resp. a plane $a$ containing $L$, and different from $l$ (Subsection 7.4. cases $\mathbf{2}$ and $\mathbf{2}^{\prime}$ ).

3. The Structure of the Ambitwistor SpaCe And the Field of $\alpha$-CONES

Conventions. Except for some results in Section 5 we will consider $\mathbf{M}$ to be a selfdual civilized 4-manifold, i.e. the (twistor) space $Z$ of $\beta$-surfaces of $\mathbf{M}$ is a Hausdorff smooth complex 3-manifold, and the projection $\mathbb{P}\left(V_{-}\right) \rightarrow Z$ is a submersion (e.g. $\mathbf{M}$ is geodesically convex); see [15].

We will frequently identify, following the deformation theory of Kodaira (see [10]), the vectors in $T_{x} \mathbf{M}$ with sections in the normal bundle $N\left(Z_{x}\right)$ of the projective line $Z_{x}$ in $Z$.

We also consider the space of null-geodesics $B$, as an open subset of $\mathbb{P}\left(T^{*} Z\right)$.

For a null-geodesic $\gamma$, resp. a $\beta$-surface $\beta \subset \mathbf{M}$, we denote by $\bar{\gamma}$, resp. $\bar{\beta}$, the corresponding point in $B$, resp. $Z$.

3.1. $\alpha$ - and $\beta$-cones on the ambitwistor space. The vectors on $B$ can be expressed in terms of infinitesimal deformations of geodesics of $\mathbf{M}$ (Jacobi fields). More precisely,

$$
T_{\bar{\gamma}} B \simeq \mathcal{J}_{\gamma}^{\perp} / \mathcal{J}_{\gamma}^{\gamma}
$$

where, for a null-geodesic $\gamma, \mathcal{J}_{\gamma}^{\perp}$ is the space of Jacobi fields $J$ such that $\nabla_{\dot{\gamma}} J \perp \dot{\gamma}$, and $\mathcal{J}_{\gamma}^{\gamma}$ is its subspace of Jacobi fields "along" $\gamma$, i.e. $J \in \mathbb{C} \dot{\gamma}$ at any point of the geodesic.

Remark. A class in $\mathcal{J}_{\gamma}^{\perp} / \mathcal{J}_{\gamma}^{\gamma}$ is represented by Jacobi fields yielding the same local section of the normal bundle $N(\gamma)$ of $\gamma$ in $\mathbf{M}$. This is equivalent to the following obvious fact:

Lemma 1. The kernel of the natural application $\mathcal{J}_{\gamma}^{\perp} \rightarrow N(\gamma)$ is $\mathcal{J}_{\gamma}^{\gamma}$.

As a consequence, Jacobi fields on $\gamma$ induce particular local sections in $N(\gamma)$, which turn out to be (conformally invariant) solutions of a differential operator of order 2 on $N(\gamma)$; see 2].

The conformal geometry of $\mathbf{M}$ induces a particular structure on $B$ : we describe it in order to obtain an expression of $W^{+}$in terms of the geometry of the (ambitwistor) space.

We have a canonical hyperplane $V_{\bar{\gamma}}$ in $T_{\bar{\gamma}} B$, defined by

$$
V_{\bar{\gamma}}:=\mathcal{J}_{\gamma}^{\perp \perp} / \mathcal{J}_{\gamma}^{\gamma}
$$

where $\mathcal{J}_{\gamma}^{\perp \perp}$ is the set of Jacobi fields $J$ everywhere orthogonal to $\dot{\gamma}$ (i.e. $\nabla_{\dot{\gamma}} J \perp \dot{\gamma}$ and $J \perp \dot{\gamma}$ ).

Now we define two fields of cones in $T B$, both contained in $V_{\bar{\gamma}}$ :

Definition 6. Let $\gamma$ be a null-geodesic in $\mathbf{M}$, and, for each point $x \in \gamma$, let $F_{x}^{\beta}$ be the $\beta$-plane containing $\dot{\gamma}_{x}$. The (infinitesimal) $\beta$-cone $V_{\bar{\gamma}}^{\beta}$ at $\bar{\gamma} \in B$ is defined as follows:

$$
V_{\bar{\gamma}}^{\beta}:=\mathcal{J}_{\gamma}^{\beta} / \mathcal{J}_{\gamma}^{\gamma} \subset \mathcal{J}_{\gamma}^{\perp \perp} / \mathcal{J}_{\gamma}^{\gamma} \simeq V_{\bar{\gamma}} \subset T_{\bar{\gamma}} B,
$$




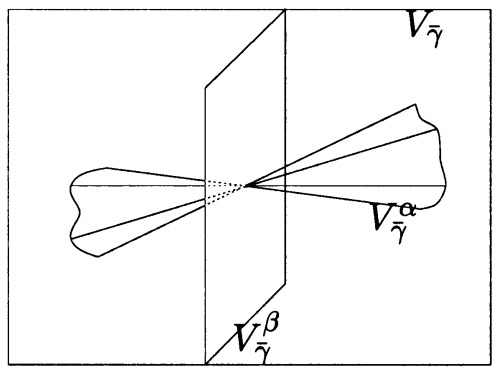

FiguRE 1.

where $\mathcal{J}_{\gamma}^{\beta}$ is the set of Jacobi fields $J$ on $\gamma$ satisfying the condition

$$
\exists x \in \gamma \text { such that } J_{x}=0 \text { and }\left(\nabla_{\dot{\gamma}} J\right)_{x} \in F_{x}^{\beta} .
$$

Proposition 3. The $\beta$-cone $V_{\bar{\gamma}}^{\beta}$ is flat, i.e. it is included in the 2-plane $F_{\bar{\gamma}}^{\beta}$ consisting of Jacobi fields contained in the $\beta$-plane defined by $\dot{\gamma}$ at each point of it.

Proof. We have to prove that $\mathcal{J}_{\gamma}^{\beta}$ is included in $\overline{\mathcal{J}}_{\gamma}^{\beta}$, defined as follows:

$$
\overline{\mathcal{J}}_{\gamma}^{\beta}:=\left\{J \text { a Jacobi field on } \gamma \mid J_{x}, \dot{J}_{x} \in F_{x}^{\beta}, \forall x \in \gamma\right\} .
$$

We will prove that $\mathcal{J}_{\gamma}^{\beta} \subset \overline{\mathcal{J}}_{\gamma}^{\beta}$; therefore it will follow that the latter is non-empty, and is a linear space of dimension 2 .

We denote by $K^{0}$ the parallel displacement, along $\gamma$, of a non-zero vector in $F_{x}^{\beta}$, transverse to $\dot{\gamma}$. Then $\left.K^{0} \in T \beta\right|_{\gamma} \backslash T \gamma$, because $\gamma$ is included in the totally geodesic surface $\beta$; thus we can characterize $F_{y}^{\beta}$ as the set $\left\{X \in T_{y} M \mid X \perp \dot{\gamma}, X \perp K^{0}\right\}$, for any $y \in \gamma$. We then observe that

$$
\dot{\gamma} \cdot\left\langle\dot{J}, K^{0}\right\rangle=\left\langle R(\dot{\gamma}, J) \dot{\gamma}, K^{0}\right\rangle=\left\langle R\left(\dot{\gamma}, K^{0}\right) \dot{\gamma}, J\right\rangle=k\left\langle K^{0}, J\right\rangle,
$$

because $R\left(\dot{\gamma}, K^{0}\right) \dot{\gamma}$ is in $F^{\beta}$, thus $R\left(\dot{\gamma}, K^{0}\right) \dot{\gamma}=h \dot{\gamma}+k K^{0}$. So the scalar function $\left\langle J, K^{0}\right\rangle$ satisfies a linear second order equation, and hence it it determined by its initial value and derivative. It follows then that it is identically zero; thus $J \in F^{\beta}$ everywhere, as claimed.

Another subset in $T_{\bar{\gamma}} B$ is the $\alpha$-cone $V_{\bar{\gamma}}^{\alpha}$, defined as follows:

Definition 7. Let $\gamma$ be a null-geodesic in $\mathbf{M}$, and, for each point $x \in \gamma$, let $F_{x}^{\alpha}$ be the $\alpha$-plane containing $\dot{\gamma}_{x}$. The (infinitesimal) $\alpha$-cone $V_{\bar{\gamma}}^{\alpha}$ at $\bar{\gamma} \in B$ is defined as follows:

$$
V_{\bar{\gamma}}^{\alpha}:=\mathcal{J}_{\gamma}^{\alpha} / \mathcal{J}_{\gamma}^{\gamma} \subset \mathcal{J}_{\gamma}^{\perp \perp} / \mathcal{J}_{\gamma}^{\gamma} \simeq V_{\bar{\gamma}} \subset T_{\bar{\gamma}} B
$$

where $\mathcal{J}_{\gamma}^{\alpha}$ is the set of Jacobi fields $J$ on $\gamma$ satisfying the condition

$$
\exists x \in \gamma \text { such that } J_{x}=0 \text { and }\left(\nabla_{\dot{\gamma}} J\right)_{x} \in F_{x}^{\alpha} .
$$

It is important to note that, in general, the projective curves $\mathbb{P}\left(V_{\bar{\gamma}}^{\alpha}\right)$ and $\mathbb{P}\left(V_{\bar{\gamma}}^{\beta}\right)$ are non-compact, as each of them corresponds to the set of points on $\gamma$, which is non-compact, in general. The field of $\alpha$-cones on $B$ is the object of main interest in this paper. We may already guess that its flatness (i.e. the situation when $V_{\bar{\gamma}}^{\alpha}$ is a subset in a 2-plane) can be related to some vanishing property of the self-dual Weyl tensor of M. See Figure 1. 
Remark. We have seen that $V_{\bar{\gamma}}^{\beta}$ is included in the 2-plane $F_{\bar{\gamma}}^{\beta}$, i.e. the condition $J_{x}=0, \dot{J}_{x} \in F_{x}$ can be generalized to the linear condition $J, \dot{J} \in F^{\beta}$, but there is no canonical way of supplying the "missing" points of $\gamma$ with some appropriate Jacobi fields in order to "complete" $V_{\bar{\gamma}}^{\alpha}$ as in the $\beta$-cones case. This would be possible, for example, if $\mathbb{P}\left(V_{\bar{\gamma}}^{\alpha}\right)$ were an open subset in a projective line. But the failure of $V_{\bar{\gamma}}^{\alpha}$ to be part of a 2-plane is measured by its projective curvature, and we will see in Section 4 that the vanishing of the latter implies the vanishing of $W^{+}$(Theorem 1).

3.2. Integral $\alpha$-cones in $Z$ and $B$. Now we study the field of $\alpha$-cones of $B$ in relation with $Z$ and the canonical projection $\pi: B \rightarrow Z$. First, we note that there are complex projective lines in $B$ tangent to the directions in $V_{\bar{\gamma}}^{\alpha}$ :

Definition 8. Let $\bar{\gamma} \in B$, and let $x \in \gamma$ be a point on the null-geodesic $\gamma$; let $F_{x}^{\alpha}$ be the $\alpha$-plane tangent to $T_{x} \gamma$. The rational curve $B_{\bar{\gamma}, x}^{\alpha}$ in $B$ (containing $\bar{\gamma}$ ) is by definition the set of null-geodesics passing through $x$ and tangent to $F_{x}^{\alpha}$.

The curves $B_{\bar{\gamma}, x}^{\alpha}, x \in \gamma$, are projected by $\pi$ onto the complex lines $Z_{x}$ through $\bar{\beta}$ (corresponding to the $\beta$-surface $\beta$ containing $\gamma$ ) tangent to the 2-plane $F^{\gamma}$.

On the other hand, it is easy to see that the complex projective lines $B_{\bar{\gamma}, x}^{\beta}$ (defined analogously to $B_{\bar{\gamma}, x}^{\alpha}$ ), which are tangent to (an open set of the directions of) $V_{\bar{\gamma}}^{\beta}$, are contained in the fibers of $\pi$. In fact, they coincide with some of the projective lines passing through the point $\gamma \in \mathbb{P}\left(T_{\bar{\beta}}^{*} Z\right) \simeq \mathbb{C P}^{2}$.

Definition 9. The integral $\alpha$-cones in $B$, resp. $Z$, are defined by:

$$
B_{\bar{\gamma}}^{\alpha}:=\bigcup_{x \in \gamma} B_{\bar{\gamma}, x}^{\alpha}(\beta \text {-cone in } B) ; \quad Z^{\gamma}:=\bigcup_{x \in \gamma} Z_{x}(\beta \text {-cone in } Z) .
$$

We intend to prove that $B_{\bar{\gamma}}^{\alpha}$ is the canonical lift of $Z^{\gamma}$ (see Proposition (5)). We know that $\pi\left(B_{\bar{\gamma}}^{\alpha}\right)=Z^{\gamma}$. We have

Proposition 4. Except for the vertices $\bar{\gamma} \in B_{\bar{\gamma}}^{\alpha}$ and $\bar{\beta} \in Z^{\gamma}$, the two integral cones $B_{\bar{\gamma}}^{\alpha}$ and $Z^{\gamma}$ are smooth, immersed surfaces of $B$, resp. $Z$.

Proof. The open set $B$ of $\mathbb{P}\left(T^{*} Z\right)$ which is the space of null-geodesics of $\mathbf{M}$ can be viewed as the space of integral curves of the geodesic distribution $G$ of lines in $\mathbb{P}(C)$, the total space of the fibre bundle of isotropic directions in $T \mathrm{M} . G_{v}$ is defined as the horizontal lift (for the Levi-Civita connection on $\mathbf{M}$ ) of $v$, which is an isotropic line in $T_{x} \mathbf{M}$. This definition is independent of the chosen metric and connection [15], and, by integrating this distribution (as $\mathbf{M}$ is civilized), we get a holomorphic map $p: \mathbb{P}(C) \rightarrow B$, where $B$ is the space of leaves of this foliation. This map can be used to compute the normal bundle of $B_{\bar{\gamma}, x}^{\alpha}, N\left(B_{\bar{\gamma}, x}^{\alpha}\right)$; see [12, 11], [15.

Indeed, we have lines $C_{\gamma, x}^{\alpha} \in \mathbb{P}(C)_{x}$, such that $\dot{\gamma}_{x} \in C_{\gamma, x}^{\alpha}$, which project onto $B_{\bar{\gamma}, x}^{\alpha}$; thus we get the following exact sequence of normal bundles:

$$
0 \rightarrow N\left(C_{\gamma, x}^{\alpha} ; p^{-1}\left(B_{\bar{\gamma}, x}^{\alpha}\right)\right) \rightarrow N\left(C_{\gamma, x}^{\alpha} ; \mathbb{P}(C)\right) \rightarrow N\left(B_{\bar{\gamma}, x}^{\alpha} ; B\right) \rightarrow 0,
$$

where we have written the ambient spaces of the normal bundles on the second position. The central bundle is trivial $\left(C_{\gamma, x}^{\alpha}\right.$ is trivially embedded in $\mathbb{P}(C)_{x} \simeq$ $\mathbb{C P}^{1} \times \mathbb{C P}^{1}$, which is trivially embedded in $\mathbb{P}(C)$ as a fibre), and it is easy to check that the left-hand bundle is isomorphic to the tautological bundle over $\mathbb{C P}^{1}, \mathcal{O}(-1)$. This proves that $N\left(B_{\bar{\gamma}, x}^{\alpha} ; B\right) \simeq \mathcal{O}(0) \oplus \mathcal{O}(0) \oplus \mathcal{O}(1)$; in particular, the conditions 
in the completeness theorem of Kodaira [10] are satisfied. Thus the lines in the integral $\alpha$-cone $B_{\bar{\gamma}}^{\alpha}$ form an analytic subfamily of the family $\left\{B_{\bar{\gamma}, x}^{\alpha}\right\}_{\bar{\gamma} \in B, x \in \gamma \subset M}$ that correspond to the sections of the normal bundle of $B_{\bar{\gamma}, x}^{\alpha}$ vanishing at $\bar{\gamma} \in B$, or, equivalently, to the points $x$ of $\gamma \subset \mathbf{M}$.

But, in order to prove the smoothness of $B_{\bar{\gamma}}^{\alpha} \backslash\{\bar{\gamma}\}$, we first remark that the surface $C_{\gamma}^{\alpha} \subset \mathbb{P}(C)$, defined as follows, is smooth:

$$
C_{\gamma}^{\alpha}:=\left\{v \in \mathbb{P}(C)_{x} \mid x \in \gamma, v \subset F_{\gamma}^{\alpha}\right\},
$$

where $F_{\gamma}^{\alpha}$ is the $\alpha$-plane containing $\dot{\gamma} . C_{\gamma}^{\alpha}$ is smooth, and $p\left(C_{\gamma}^{\alpha}\right)=B_{\bar{\gamma}}^{\alpha}$. We note now that $C_{\gamma}^{\alpha}$ is everywhere, except at the points of $p^{-1}(\bar{\gamma})$, transverse to the fibers of the submersion $p: \mathbb{P}(C) \rightarrow B$. We may conclude that $B_{\bar{\gamma}}^{\alpha} \backslash\{\gamma\}$ is a smooth analytic submanifold of $B$ (not closed).

We can use similar methods to prove that $Z^{\gamma} \backslash\{\bar{\beta}\}$ is an immersed submanifold of $Z$ (by using the projection $\pi: B \rightarrow Z$ ).

There is another argument for this latter claim, which gives the tangent space to $Z^{\gamma}$ at any point.

We see $Z^{\gamma}$ as the trajectory of a 1-parameter deformation of $Z_{x}$ : we fix $\bar{\beta}$ and we "turn" $Z_{x}$ around $\bar{\beta}$ by keeping it tangent to $F^{\gamma}$. The trajectory of this deformation is smooth in $\zeta \in Z^{\gamma} \backslash \beta$ iff any non-identically-zero section $\nu$ of the normal bundle $N\left(Z_{x}\right)$ corresponding to this 1-parameter deformation does not vanish at $\zeta$. In particular, the tangent space $T_{\zeta} Z^{\gamma}$ is spanned by $T_{\zeta} Z_{x}$ and $\nu(\zeta)$.

But the sections $\nu$ generating this deformation are the sections of $N\left(Z_{x}\right)$ vanishing at $\bar{\beta}$, and they vanish at only one point (and even there, only to order 0 ) unless they are identically zero, because $N\left(Z_{x}\right) \simeq \mathcal{O}(1) \oplus \mathcal{O}(1)$.

Remark. The values of these sections at the points of $Z_{x}$ other than $\bar{\beta}$, plus their derivatives at $\bar{\beta}$ (well-defined as they all vanish at $\bar{\beta}$ ), define a 1-dimensional subbundle of $N\left(Z_{x}\right)$ which is isomorphic to $\mathcal{O}(1)$. In fact, we have a 1-1 correspondence between the subbundles of $N\left(Z_{x}\right)$ isomorphic to $\mathcal{O}(1)$ and the 2-planes in $T_{\bar{\beta}} Z$. Then, the space of holomorphic sections of such a bundle is a linear space of dimension 2, consisting of a family of sections of $N\left(Z_{x}\right)$ vanishing on different points of $Z_{x}$. Thus we get a 2-plane $F^{\alpha}$ of isotropic vectors in $T_{x} \mathbf{M}$, which is easily seen to be an $\alpha$-plane, as the $\beta$-plane $F_{x}^{\beta}=T_{x} \beta$ consists of the set of all sections of $N\left(Z_{x}\right)$ vanishing at $\bar{\beta}$ (we have $F_{x}^{\alpha} \cap T_{x} \beta=T_{x} \gamma$ ). The tangent space to $Z^{\gamma}$ at a point $\zeta \in Z_{x}$ is spanned by the subbundle of $N\left(Z_{x}\right)$ (isomorphic to $\mathcal{O}(1)$ - see above) defined by the isotropic vectors $v \in F_{x}^{\alpha}$. If $\gamma^{\zeta}$ is the null-geodesic generated by $v^{\zeta}$, we conclude that $T_{\zeta} Z^{\gamma}$ is the 2-plane determined by $\gamma^{\zeta}$, and that $\zeta=\pi\left(\overline{\gamma^{\zeta}}\right)$. See Figure 2.

Example. If $\mathbf{M}=\mathbb{P}(E) \times \mathbb{P}(E)^{*} \backslash \mathcal{F}$, then the integral $\alpha$-cone $Z^{\gamma}$ in $Z$, for $\gamma \equiv$ $F^{\gamma}=F^{\varphi} \subset T_{(L, l)} Z$ (where $\varphi: \mathbb{P}(l) \rightarrow \mathbb{P}\left(L^{o}\right)$ is a projective diffeomorphism), is the (smooth away from the vertex $(L, l))$ surface $\{(S, \varphi(s)) \mid S \neq L, s \neq l, \varphi(s \cap l)=S\}$. Its compactification (by adding the special cycle $\bar{Z}_{(L, l)}$ ) is singular (Subsection 7.4).

As any smooth surface in $Z$ has a canonical lift in $B=\mathbb{P}\left(T^{*} Z\right)$, we get

Proposition 5. The integral $\alpha$-cone $B_{\bar{\gamma}}^{\alpha}$ is the canonical lift of the integral $\alpha$-cone $Z^{\gamma}$ on $Z$. See Figure 3 . 


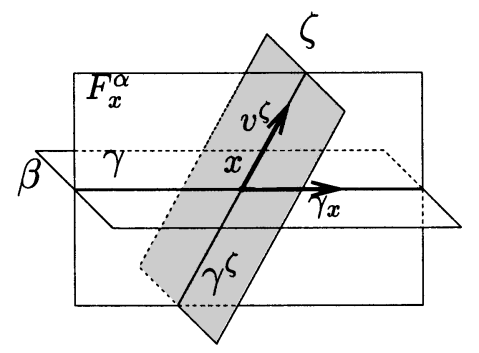

FIGURE 2.

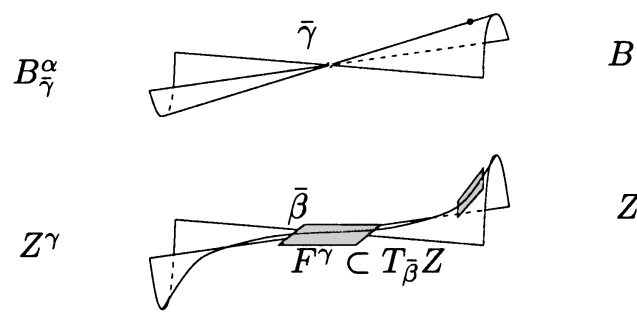

FiguRE 3.

Remark. Basically, this lift can only be defined for $Z^{\gamma} \backslash\{\bar{\beta}\}$, but in this special case it can be extended by continuity to $\bar{\beta}$. Of course, the smoothness of the lifted surface can only be deduced away from the vertex $\bar{\gamma}$ (from the smoothness of $\left.Z^{\gamma} \backslash\{\bar{\beta}\}\right)$.

\section{The projective Curvature of the $\alpha$-CONe $V_{\gamma}^{\alpha}$ AND THE SELF-DUAL WEYL TENSOR $W^{+}$ON M}

As noted in Section 3, we intend to find a relation between the "curvature" of the $\alpha$-cone $V_{\bar{\gamma}}^{\alpha}$ (its non-flatness) and the Weyl tensor $W^{+}$of $(\mathbf{M}, c)$. We begin by defining the projective curvature of $V_{\gamma}^{\alpha}$ : A projective structure on a manifold $X$ is an equivalence class of linear connections yielding the same geodesics. In such a space, we can define the projective curvature of a curve $S$ at a point $\sigma$ as the linear application $k: T_{\sigma} S \otimes T_{\sigma} S \rightarrow N(S)_{\sigma}=T_{\sigma} X / T_{\sigma} S$, with $k(Y):=\nabla_{Y} Y$ (modulo $T_{\sigma} S$ ), for $\nabla$ any connection in the projective structure of $X$. In particular, we take for $X$ the projective space $\mathbb{P}\left(T_{\bar{\gamma}} B\right)$, with its canonical projective structure, and for $S$ we take $\mathbb{P}\left(V_{\bar{\gamma}}^{\alpha}\right)$, the projectivized $\alpha$-cone in $\bar{\gamma}$.

Definition 10. The projective curvature of the $\alpha$-cone $V_{\bar{\gamma}}^{\alpha}$ at the generating line $\sigma \subset V_{\bar{\gamma}}^{\alpha}$ is the projective curvature of $S:=\mathbb{P}\left(V_{\bar{\gamma}}^{\alpha}\right)$ in $\sigma$, and is identified with a linear application

$$
K_{\gamma, x}^{\alpha}: T_{\sigma} S \otimes T_{\sigma} S \rightarrow N(S)_{\sigma},
$$

where $\sigma$ is the tangent direction to $B_{\bar{\gamma}, x}^{\alpha}$ in $\bar{\gamma}$.

In order to compute the projective curvature of $V_{\bar{\gamma}}^{\alpha}$, we first establish some canonical isomorphisms between the spaces appearing in the above definition and some linear subspaces of $T_{x} \mathbf{M}$. We fix the geodesic $\gamma$, the point $x \in \gamma$ (therefore 
also $\left.\sigma=T_{\bar{\gamma}} B_{\bar{\gamma}, x}^{\alpha} \in \mathbb{P}\left(T_{\bar{\gamma}} B\right)\right)$, and, thus, the $\alpha$-plane $F_{x}^{\alpha} \subset T_{x} \mathbf{M}$ containing $\dot{\gamma}_{x}$, as well as $\dot{\gamma}_{x}^{\perp} \subset T_{x} \mathbf{M}$, the space orthogonal to $\dot{\gamma}_{x}$.

For simplicity, in the following lemmas we will omit some indices referring to these fixed objects.

Lemma 2. There is a canonical isomorphism $\tau$ between the tangent space $T_{\sigma} S$ to the projective cone $S=\mathbb{P}\left(V_{\bar{\gamma}}^{\alpha}\right)$ and the tangent space $T_{x} \gamma$ to the geodesic $\gamma$ at the point $x$ corresponding to the direction $\sigma \in \mathbb{P}\left(T_{\bar{\gamma}} B\right)$.

Proof. Let $Y \in T_{x} \gamma$. We define $\tau^{-1}(Y)$ as follows. Recall that $T_{\sigma} S \simeq \operatorname{Hom}(\sigma, E / \sigma)$, where $E\left(=E_{x}\right):=T_{\sigma} V_{\bar{\gamma}}^{\alpha}$ (the tangent space at a point to a cone is the same for all points on the line containing the point). We know that $\sigma$ corresponds to $\mathcal{J}_{\gamma, x}^{\alpha}$, the space of Jacobi fields on $\gamma$ vanishing at $x$ and such that $\dot{J}_{x} \in F^{\alpha}$. It will be shown in the proof of the next theorem that $E$ consists of classes of Jacobi vector fields such that $J_{x}, \dot{J}_{x} \in F^{\alpha}$, (4).

Then, on a representative Jacobi field $J \in \mathcal{J}_{\gamma, x}^{\alpha}$, we define $\tau^{-1}(Y)$ to be the class of Jacobi fields in $E / \sigma$ represented by the Jacobi field $J^{Y}$ on $\gamma$ which is given by $J_{x}^{Y}:=\nabla_{Y} J, \dot{J}_{x}^{Y}:=0$. We remark that $\nabla_{Y} J$ is what we usually denote $\dot{J}$, when the parameter on $\gamma$ is understood.

It is straightforward to check that $J \mapsto J^{Y}$ induces an isomorphism $\tau^{-1}(Y)$ : $\sigma \rightarrow E / \sigma$ for each non-zero $J \in \sigma=\mathcal{J}_{\gamma, x}^{\alpha} / \mathcal{J}_{\gamma}^{\gamma}$.

We remark that $V_{\bar{\gamma}}^{\alpha} \subset V_{\bar{\gamma}}$, the 4-dimensional subspace represented by Jacobi fields $J$ such that $J, \dot{J} \perp \dot{\gamma}$. We further introduce the subspace $H_{\bar{\gamma}, x}^{\alpha} \subset V_{\bar{\gamma}}$, represented by Jacobi fields $J$ as before, with the additional condition $J_{x} \in F_{x}^{\alpha}$. It is a 3-dimensional subspace, and it contains $E_{x}$. The curvature of $V_{\bar{\gamma}}^{\alpha}$ will take values in

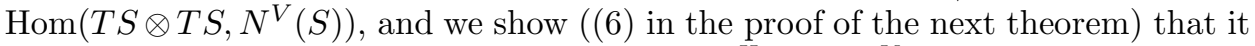
takes values in a smaller space, $\operatorname{Hom}\left(T S \otimes T S, N^{H}(S)\right) . N_{\sigma}^{V}(S) \simeq \operatorname{Hom}\left(\sigma, V_{\bar{\gamma}}\right) / T_{\sigma} S$ is just the normal space of $S$ in $\mathbb{P}\left(V_{\gamma}\right)$ at $\sigma$, and $N^{H}(S)$ is the subspace of $N_{\sigma}^{V}(S)$ consisting of elements represented by $\xi \in \operatorname{Hom}\left(\sigma, H_{\bar{\gamma}, x}^{\alpha}\right) \subset \operatorname{Hom}\left(\sigma, V_{\bar{\gamma}}\right)$.

Lemma 3. There is a canonical isomorphism

$$
\rho: N^{H}(S) \rightarrow \operatorname{Hom}\left(F^{\alpha} / T \gamma, \gamma^{\perp} / F^{\alpha}\right) .
$$

Proof. As $H$ is a subbundle of the normal bundle $N(S), N^{H}(S)$ is isomorphic to $\operatorname{Hom}(\sigma, H / E)$. As in Lemma 2, we will construct the inverse isomorphism $\rho^{-1}$. Let $\xi: F^{\alpha} / T \gamma \rightarrow \gamma^{\perp} / F^{\alpha}$ be a linear application. Let $\xi_{0}: F^{\alpha} \rightarrow \gamma^{\perp}$ be a representant of $\xi$ (it involves a choice of a complementary space to $F^{\alpha}$ in $\gamma^{\perp}$ ). We define $\rho^{-1}(\xi) \in \operatorname{Hom}(\sigma, H / E)$ as being induced by the following linear application between spaces of Jacobi fields on $\gamma$.

$\rho^{-1}(\xi): \mathcal{J}_{\gamma, x}^{\alpha} \rightarrow \mathcal{J}_{\gamma, x}^{\alpha, \perp}$, where the second space corresponds to $H_{x}$, i.e. it contains Jacobi fields $J$ such that $J_{x} \in F^{\alpha}, \dot{J}_{x} \perp \dot{\gamma}_{x}$. Consider a parameterization of $\gamma$ around $x$, and let $J \in \mathcal{J}_{\gamma, x}^{\alpha}$. We define $J^{\xi}:=\rho^{-1}(\xi)(J)$ by $J_{x}^{\xi}:=0, \dot{J}_{x}^{\xi}:=\xi_{0}\left(\dot{J}_{x}\right)$, and it is easy to check that the class of $J^{\xi}$ in $H / E$ is independent of the representant $\xi_{0}$ such that $\rho^{-1}$ is well-defined. It is also obviously invertible.

We are now in position to translate the projective curvature of $V_{\gamma}^{\alpha}$ in terms of conformal invariants of $(\mathbf{M}, c)$. 
Theorem 1. Let $x$ be a point on a null-geodesic $\gamma$. Then the projective curvature $K$ of the $\alpha$-cone $V_{\bar{\gamma}}^{\alpha}$ at $\sigma$ (corresponding to $x$, see Definition 10 ), which is a linear map

$$
K: T_{\sigma} S \otimes T_{\sigma} S \rightarrow N^{V}(S)_{\sigma},
$$

takes values in $N^{H}(S)_{\sigma}$ (see above), and is canonically identified with the linear map

$$
K^{\prime}: T_{x} \gamma \otimes T_{x} \gamma \rightarrow \operatorname{Hom}\left(F_{x}^{\alpha} / T_{x} \gamma, \gamma_{x}^{\perp} / F_{x}^{\alpha}\right)
$$

defined by the self-dual Weyl tensor of $\mathbf{M}$ :

$$
K^{\prime}(Y, Y)(X)=W^{+}(Y, X) Y, \quad Y \in T_{x} \gamma, X \in F_{x}^{\alpha} .
$$

Proof. Consider the following analytic map, which parameterizes, locally around $x \in \gamma$, the deformations of the geodesic $\gamma$ that correspond to points contained in the integral $\alpha$-cone $B_{\bar{\gamma}}^{\alpha}$ :

$$
f: U \rightarrow \mathbf{M}, f(t, s, u)=\gamma^{t, s}(u),
$$

where $U$ is a neighborhood of the origin in $\mathbb{C}^{3}$, and $\gamma^{t, s}$ is a deformation of the null-geodesic $\gamma$, such that

$$
\gamma^{t, s}(t)=\gamma(t), \dot{\gamma}^{t, s}(t) \in F_{\gamma(t)}^{\alpha},
$$

where the parameterization of the geodesic $\gamma$ satisfies $\gamma(0)=x$, and $F_{\gamma(u)}^{\alpha}$ is the $\alpha$-plane in $T_{\gamma(u)} \mathbf{M}$ containing $\dot{\gamma}(u)$.

Convention. We know that $f$ is defined around the origin in $\mathbb{C}^{3}$, so there exists a polydisc centered at the origin included in $U$, and so all the relations that we will use are true for values of the variables $t, s, u$ sufficiently close to 0 . For simplicity, we will not mention these domains.

The geodesics $\gamma^{t, s}$ correspond to points in $B_{\bar{\gamma}, \gamma(t)}^{\alpha}$, and the Jacobi fields $J^{t}$ on $\gamma$, defined as

$$
J^{t}(u):=\partial_{s} f(t, 0, u) \in T_{\gamma(u)} \mathbf{M},
$$

correspond to vectors in $V_{\bar{\gamma}}^{\alpha}$ tangent to the above-mentioned lines. We suppose that the deformation $f$ is effective, i.e. $\partial_{u} \gamma^{t, s}(u) \neq 0$ and $J^{t} \notin \mathcal{J}_{\gamma}^{\gamma}$, which is equivalent to $\dot{J}^{t}(t) \notin T_{\gamma(t)} \gamma$. In order to compute the projective curvature of $V_{\bar{\gamma}}^{\alpha}$, we thus need to study the (second order) infinitesimal variation of these Jacobi fields on $\gamma$. As they are determined by their value and first order derivative in $\gamma(0)=x$, we need to evaluate $\left.\partial_{t} J^{t}(0)\right|_{t=o},\left.\partial_{t} \dot{J}^{t}(0)\right|_{t=0}$ for the first derivative of $J^{t}$ at $t=0$, and $\left.\partial_{t}^{2} J^{t}(0)\right|_{t=0},\left.\partial_{t}^{2} \nabla \dot{J}^{t}(0)\right|_{t=0}$ for the second. Dots mean, as before, covariant differentiation with respect to the "speed" vector $\dot{\gamma}$; thus they correspond to the operator $\partial_{u}$.

As the covariant derivation $\nabla$ has no torsion, we can apply the usual commutativity relations between the operators $\partial_{t}, \partial_{s}, \partial_{t}$ and use them to differentiate the following equation, which follows directly from the definition of $f$ and $J^{t}$ :

$$
J^{t}(t)=0 \forall t
$$

We get then

$$
\partial_{t} J^{t}(t)+\dot{J}^{t}(t)=0
$$


We recall now that, besides (10), we have $\dot{J}^{t}(t) \in F_{\gamma(t)}^{\alpha}$; thus $\dot{J}^{t}(t)$ is isotropic, which implies that

$$
\left\langle\partial_{t} \dot{J}^{t}(t), \dot{J}^{t}(t)\right\rangle=0
$$

as $\ddot{J}^{t}(t)=R\left(\dot{\gamma}(t), J^{t}(t)\right) \dot{\gamma}(t)=0$. Equations (2) and (3) prove that

$$
\left.\partial_{t} J^{t}\right|_{t=0} \in \mathcal{J}_{\gamma, x}^{\alpha}
$$

which completes the proof of Lemma 2. From (3), it equally follows that $\partial_{t} \dot{J}^{t}(t)$ is isotropic, and, by differentiating (3), we get

$$
\left\langle\partial_{t}^{2} \dot{J}^{t}(t), \dot{J}^{t}(t)\right\rangle=-\left\langle\partial_{t} \ddot{J}^{t}(t), \dot{J}^{t}(t)\right\rangle \text {. }
$$

From (2) we have that $\partial_{t} J^{t}(t)$ is isotropic, and also

$$
\partial_{t}^{2} J^{t}(t)+2 \partial_{t} \dot{J}^{t}(t)=0
$$

which, together with (3), implies that $\left.\partial_{t}^{2} J^{t}(0)\right|_{t=0} \in F_{x}^{\alpha}$. Then we have

$$
\left.\partial_{t}^{2} J^{t}\right|_{t=0} \in \mathcal{J}_{\gamma, x}^{\alpha, \perp}
$$

which proves that the curvature $K$ of the $\alpha$-cone takes values in $N^{H}(S)$, as it is represented by $\left.\partial_{t}^{2} J^{t}\right|_{t=0}$.

In view of Lemmas 2 and 3 it is clear now that the projective curvature $K$ is represented by the following application:

$$
\left.(\dot{\gamma}, \dot{\gamma}, \dot{J})_{x} \longmapsto \partial_{t}^{2} J^{t}(0)\right|_{t=0} .
$$

From (5), as $\partial_{t} \ddot{J}^{t}(t)=R\left(\dot{\gamma}, \partial_{t} J^{t}\right) \dot{\gamma}$ and $\partial_{t} J^{t}(t)=-\dot{J}^{t}(t)$, we get

$$
\langle K(\dot{\gamma}, \dot{\gamma})(\dot{J}), \dot{J}\rangle=\langle R(\dot{\gamma}, \dot{J}) \dot{\gamma}, \dot{J}\rangle \text {. }
$$

The right-hand side actually involves only $W^{+}$, as the other components of the Riemannian curvature vanish on this combination of vectors. Thus we can replace $R$ by $W^{+}$in the above relation. On the other hand, the class of $W^{+}(\dot{\gamma}, \dot{J}) \dot{\gamma}$ modulo $F^{\alpha}$ is determined by its scalar product with $\dot{J}$, which represents a non-zero generator of $F^{\alpha} / T \gamma$.

The proof of the theorem is now complete.

Remark. We may ask whether the projective lines in $Z$ are the geodesics of some projective structure. Indeed, in the conformally flat case, when $\mathbf{M}$ is the Grassmannian of 2-planes in $\mathbb{C}^{4}$ (the complexification of the Möbius 4 -sphere), $Z \simeq \mathbb{C P}^{3}$, and the complex lines are given by the standard (flat) projective structure. But there are two reasons (related to each other, as we will soon see) why $Z$ cannot carry, in general, a canonical projective structure. First, we do not necessarily have projective lines $Z_{x} \ni \bar{\beta}$ in every direction of $T_{\bar{\beta}} Z$ (this would mean that $\beta \simeq \mathbb{C P}^{2}$, see the next section for a treatment of this problem), and second, the lift of a 2-plane $F^{\gamma} \subset T_{\bar{\beta}} Z$ would be a 2-plane in $T_{\bar{\gamma}} B$, so $V_{\bar{\gamma}}^{\alpha}$ would be a flat cone.

Corollary 1. The projective lines $Z_{x}$ in the twistor space $Z$ are geodesics of a projective structure iff it is projectively flat and $\mathbf{M}$ is conformally flat.

Proof. If $Z$ admits a projective structure, some of whose geodesics are the lines $Z_{x}$, then we have, for a fixed $\bar{\beta} \in Z$, a linear connection around $\bar{\beta}$, whose geodesics in the directions of $Z_{x}, \bar{\beta} \in Z_{x}(\Leftrightarrow x \in \beta \subset \mathbf{M})$ coincide, locally, with $Z_{x}$. This means that the integral $\alpha$-cone $Z^{\gamma}$, for $\gamma \subset \beta$ a null-geodesic, is part of a complex surface (namely $\exp \left(F^{\gamma}\right)$, where $F^{\gamma} \subset T_{\bar{\beta}} Z$ is the 2-plane corresponding to $\gamma$ ). Then the 
integral $\alpha$-cone $B_{\bar{\gamma}}^{\alpha}$, the lift to $B$ of $Z^{\gamma}$, is also a complex surface, and so $V_{\bar{\gamma}}^{\alpha}$ is a subset of the tangent space $T_{\bar{\gamma}} B_{\bar{\gamma}}^{\alpha}$, thus a flat cone. As this is true for all points of $Z$ and for all null-geodesics $\gamma$, Theorem 1 implies that $\mathbf{M}$ is flat.

On the other hand, it is well-known that the twistor space of a conformally flat manifold admits a flat projective structure, for which the projective lines $Z_{x}$ are geodesics, [1].

\section{COMPACtNess OF NULL-GEODESICS AND CONFORMAL FlatNesS}

5.1. Complete $\alpha$-cones in $Z$. We have given, in the preceding section, a way to measure the projective curvature of the $\alpha$-cone in $B$; we shall see now what happens in the special case when this cone is complete at a point $\bar{\gamma}$, i.e. when $\mathbb{P}\left(V_{\bar{\gamma}}^{\alpha}\right)$ is a compact submanifold in $\mathbb{P}\left(T_{\gamma} B\right)$.

This situation appears for example if, for every direction in $F^{\gamma} \subset T_{\bar{\beta}} Z$, there are projective lines in $Z$ tangent to it.

Theorem 2. Let $Z$ be the twistor space of the connected civilized self-dual 4manifold $(\mathbf{M}, c)$, and suppose that, for a point $\beta \in Z$ and for a 2-plane $F^{\alpha} \subset T_{\bar{\beta}} Z$, there are projective lines $Z_{x}$ tangent to each direction of $F^{\alpha}$. Then $(\mathbf{M}, c)$ is conformally flat.

Proof. The idea is to prove that the integral $\alpha$-cone $Z^{\gamma}$ is a smooth surface. We know that this holds at all its points except the vertex $\bar{\beta}$ (Proposition 4). The fact that all directions in $F^{\gamma}$ admit a tangent line is a necessary condition for this cone to be a smooth surface, as it needs to be well-defined around $\bar{\beta}$.

We choose an auxiliary Hermitian (real) metric $h$ on $Z$. Its restrictions $h_{x}$ to the lines $Z_{x} \subset Z^{\gamma}$ yield Kählerian metrics on these lines; in fact these metrics are deformations of one another, just like the lines $Z_{x}$ are. This means that the metrics $h_{x}$ depend continuously on $x \in \mathbb{P}\left(F^{\alpha}\right)$, a parameter in a compact set. We can therefore find a lower bound $r_{0}>0$ for the injectivity radius of all $\left(Z_{x}, h_{x}\right)$ at $\bar{\beta}$, and a finite upper bound $R$ for the norm of all the second fundamental forms $H_{x}: T Z_{x} \otimes T Z_{x} \rightarrow\left(T Z_{x}\right)^{\perp}(\subset T Z)$. We can also suppose that $r_{0}$ is smaller than the injectivity radius of $(Z, h)$ at $\bar{\beta}$.

The first step is to prove that $Z^{\gamma}$ is a submanifold of class $\mathcal{C}^{1}$. As its tangent space is everywhere a complex subspace of $T Z$, it will follow that it is a complex analytic submanifold.

Consider now the exponential map $\exp _{\bar{\beta}}: T_{\bar{\beta}} Z \rightarrow Z$, defined for the metric $h$. If we restrict it to a ball of radius less than $r_{0}$, it is a diffeomorphism into $Z$. The image of the complex plane $F^{\alpha}$ is then a smooth 4-dimensional real submanifold $S$ of $Z$, and there exists a positive number $r_{1}$ such that the exponential map in the directions normal to $S$,

$$
\exp _{S}: T S^{\perp} \rightarrow Z, \exp (Y):=\exp _{y}(Y), y \in S, Y \in T_{y} S^{\perp},
$$

restricted to the vectors of length less than $r_{1}$, is a diffeomorphism.

The image of this diffeomorphism is a tubular neighborhood of $S$, and we will denote by $N(S, r)$ such a tubular neighborhood of "width" $r$, for $r<r_{1}$.

The existence of an upper bound $R$ for the second fundamental forms of $Z_{x}, \forall x \in$ $\gamma$, implies the following fact. 
Lemma 4. For any $r<r_{1}$, there is a neighborhood $U \subset T_{\bar{\beta}} Z$ of the origin such that $\exp (U) \cap Z^{\gamma}$ is contained in $N(S, r)$ and is transverse to the fibers of the orthogonal projection $p^{S}: N(S, r) \rightarrow S, p^{S}(\exp (Y)):=y$, where $Y \in T_{y} S$.

This is standard if $Z^{\gamma}$ is a submanifold; but it is also true in our case, where $Z^{\gamma}$ is a union of submanifolds $Z_{x}$.

Now it is easy to prove that $Z^{\gamma}$ is a $\mathcal{C}^{1}$ submanifold of $Z$ (the projection $p^{S}$ yields a local $\mathcal{C}^{1}$ diffeomorphism from a neighborhood of $\bar{\beta}$ in $S$ to a neighborhood of $\bar{\beta}$ in $Z^{\gamma}$; it is $\mathcal{C}^{1}$ at $\bar{\beta}$ because $S$ is tangent to $Z^{\gamma}$ at $\bar{\beta}$ ).

So $Z^{\gamma}$ is a $\mathcal{C}^{1}$ submanifold of $Z$. Its tangent space is complex at each point, and so $Z^{\gamma}$ is a complex-analytic surface immersed in $Z$.

Then $B_{\bar{\gamma}}^{\alpha} \subset B=\mathbb{P}\left(T^{*} Z\right)$, being the lift of $Z^{\gamma}$, is a smooth analytic surface immersed in $B$; in particular, the $\alpha$-cone $V_{\bar{\gamma}}^{\alpha}$ is a complex plane.

Theorem 1 implies that $W^{+}$vanishes on the $\alpha$-plane $F_{x}^{\alpha} \subset T_{x} \mathbf{M}$ which contains $\dot{\gamma}_{x}$, for every point $x \in \gamma$. Now, the plane $F^{\gamma} \subset T_{\bar{\beta}} Z$ is not the only one admitting projective lines $Z_{x}$ tangent to any of its directions: all planes "close" to $F^{\gamma}$ have the same property. Then $W^{+}$vanishes on a neighborhood of $\gamma$, hence on the whole connected manifold $\mathbf{M}$.

Remark. There is a more general situation where the integral $\alpha$-cone $Z^{\gamma}$ through $\beta$ is smooth in $\beta$.

Theorem 2. Suppose that, for each direction $\sigma \in \mathbb{P}\left(T_{\beta} Z\right)$, there is a smooth (not necessarily compact) curve $Z_{\sigma}$, tangent to $\sigma$, such that

(i) if $\sigma$ is tangent to a projective line $Z_{x}$, then $Z_{\sigma}=Z_{x}$, and

(ii) $Z_{\sigma}$ varies smoothly with $\sigma \in \mathbb{P}\left(F^{\gamma}\right)$.

Then

$$
\bar{Z}_{\beta}^{\gamma}:=\bigcup_{\sigma \in \mathbb{P}\left(F^{\gamma}\right)} Z_{\sigma}
$$

is a smooth surface around $\beta$ containing the $\alpha$-cone $Z^{\gamma}$, and $W^{+}\left(F_{x}^{\gamma}\right)=0, \forall x \in \gamma$, where $F_{x}^{\gamma} \subset T_{x} \mathbf{M}$ is the $\alpha$-plane containing $\dot{\gamma}$.

The proof is similar to that of the previous theorem. Note that, if there is a direction $\sigma$ which is not tangent to a projective line $Z_{x}$, we cannot apply the deformation argument in Theorem 2 to conclude that $W^{+}$vanishes everywhere.

Example. If $\mathbf{M}=\mathbb{P}(E) \times \mathbb{P}(E)^{*} \backslash \mathcal{F}$, then $Z=\mathcal{F}$ and there are some particular planes for which the conditions in Theorem $2^{\prime}$ are satisfied, although Theorem 2 never applies to $Z$ : for a generic 2-plane $F^{\gamma}$, the $\alpha$-cone $V_{\gamma}^{\alpha}$ is not flat. These particular planes in $T Z$ correspond to the vanishing of $W^{+}$on some particular $\alpha$-planes, but $\mathbf{M}$ is not anti-self-dual (see Section [7.3, and also 7.7, 7.8).

The following result is a direct consequence of Theorem 2;

Theorem 3. If a civilized self-dual complex 4-manifold (M,c) admits a compact null-geodesic, then the conformal structure of $\mathbf{M}$ is flat, and the null-geodesic is simply-connected.

We simply have to use the fact that a null-geodesic $\gamma$ of a civized self-dual manifold identifies with an open set of $\mathbb{P}\left(F^{\gamma}\right)$, where $F^{\gamma}$ is the associated 2-plane in $T_{\bar{\beta}} Z$, where $\beta \supset \gamma$.

The condition that $\mathbf{M}$ be civilized is not essential, if we assume that $\gamma$ is simplyconnected (and compact); in order to prove that, we need to cover $\gamma$ with civilized 
(e.g. geodesically connected) open sets $U_{i}$, and relate the local twistor spaces $Z_{i}:=Z\left(U_{i}\right)$; the key point is that, if $\gamma$ is diffeomeorphic to $\mathbb{C P}^{1}$, it turns out that a neighbourhood of $\bar{\beta} \in Z_{i}$-for $\beta$ the $\beta$-surface containing $\gamma$ - can be identified with the space of deformations of $\gamma$ as a compact curve (2], Proposition 5). Then we conclude, using the criterion from Theorem $2^{\prime}$ and a deformation argument, that $\mathbf{M}$ is conformally flat.

This method is used in 2 2 to prove the same thing starting from a conformal complex 3-manifold (using the LeBrun correspondence, i.e. the local realization of a conformal 3-manifold as the conformal infinity of a (germ-unique) self-dual manifold [12]), but we also show there, by different methods, that, in all generality, a conformal complex $n$-manifold $(n \geq 4)$ containing a compact, simply-connected null-geodesic is conformally flat ([2], Theorem 4).

\section{The Projective struCture of $\beta$-SuRfaces in a Self-DuAl Manifold}

The null-geodesics contained in a $\beta$-surface $\beta$ define a projective structure on the totally-geodesic surface $\beta$, which is also given by any connection on $\beta$ induced by a Levi-Civita connection on $\mathbf{M}$. We claim that this projective structure is flat, i.e. locally equivalent to $\mathbb{C P}^{2}$.

Example. If $\mathbf{M}=\mathbb{P}(E) \times \mathbb{P}(E)^{*} \backslash \mathcal{F}$, then a $\beta$-surface indexed by $(L, l) \in \mathcal{F}$ is $\beta^{(L, l)}=\{(A, a) \mid A \subset l, L \subset a, A \not \subset a\} \simeq \mathbb{C}^{2}$, and the null-geodesics in $\beta^{(L, l)}$ are identified with the affine lines in $\mathbb{C}^{2}$ (see Section 7.5).

To prove the projective flatness of a 2 -dimensional manifold $\beta$, we need to prove that the Thomas tensor $T$ vanishes identically 20. This tensor is an analog of the Cotton-York tensor in conformal geometry (there is also a Weyl tensor of a projective structure, but it only appears in dimensions greater than 2).

For a connection $\nabla$ in the projective class of $\beta$, the Thomas tensor is defined as follows [20]: For $X, Y, Z \in T \beta$,

$$
\begin{aligned}
T(X, Y, Z):= & -2\left(\nabla_{Z} K\right)(Y) X+2\left(\nabla_{Y} K\right)(Z) X \\
& -\left(\nabla_{Z} K\right)(X) Y+\left(\nabla_{Y} K\right)(X) Z,
\end{aligned}
$$

where the derivation involves only the curvature term $K$, which is defined by $K(Y) X:=\operatorname{tr} R(Y, \cdot) X$, the trace of the endomorphism $R(Y, \cdot) X \in \operatorname{End}(T \beta)$.

The Thomas tensor is independent of the connection $\nabla$. Therefore we will consider that $\nabla$ is induced by a Levi-Civita connection on $\mathbf{M}$.

Proposition 6. The Thomas tensor of a $\beta$-surface can be expressed in terms of the anti-self-dual Cotton-York tensor of $\mathbf{M}$. Thus it is identically zero.

Proof. First we need to define the anti-self-dual Cotton-York tensor as an irreducible component of the Cotton-York tensor of $\mathbf{M}$.

Convention. We denote by $C$ the Cotton-York tensor of $(\mathbf{M}, c)$; we will not use this letter for the isotropic cone in this section.

The Cotton-York tensor is not conformally invariant; its definition depends on a (local) metric $g$ in the conformal structure, which is supposed to be fixed [5]:

$$
C(X, Y)(Z):=\left(\nabla_{X} h\right)(Y, Z)-\left(\nabla_{Y} h\right)(X, Z), \forall X, Y, Z \in T \mathbf{M},
$$

where $h$ is the normalized Ricci tensor of $\mathbf{M}$,

$$
h=\frac{1}{2 n(n-1)} \mathrm{Scal} \cdot g+\frac{1}{n-2} \operatorname{Ric}_{0},
$$


$\mathrm{Ric}_{0}$, Scal being the trace-free Ricci tensor, resp. the scalar curvature of the metric $g$, and $n:=\operatorname{dimM}$. In our case $n=4$, but the formula applies in all dimensions greater than $2[5]$.

Remark. The Cotton-York tensor $C$ of $\mathbf{M}$ is a 2 -form with values in $T^{*} \mathbf{M}$; thus it has two components, $C^{+} \in T^{*} \mathbf{M} \otimes \Lambda^{+} \mathbf{M}$, and $C^{-} \in T^{*} \mathbf{M} \otimes \Lambda^{-} \mathbf{M}$. $C$ satisfies a first Bianchi identity, due to the fact that $h$ is a symmetric tensor, and also a contracted (second) Bianchi identity, which comes from the second Bianchi identity in Riemannian geometry, [5]:

$$
\begin{aligned}
& \sum C(X, Y)(Z)=0, \text { circular sum; } \\
& \sum C\left(X, e_{i}\right)\left(e_{i}\right)=0, \text { trace over an orthonormal basis. }
\end{aligned}
$$

That means that $C \in \Lambda^{2} \mathbf{M} \otimes \Lambda^{1} \mathbf{M}$, and is orthogonal on $\Lambda^{3} \mathbf{M} \subset \Lambda^{2} \mathbf{M} \otimes \Lambda^{1} \mathbf{M}$ and on $\Lambda^{1} \mathbf{M}$, which is identified with the image in $\Lambda^{2} \mathbf{M} \otimes \Lambda^{1} \mathbf{M}$ by the metric adjoint of the contraction (11).

Now, the Hodge operator $*: \Lambda^{2} \mathbf{M} \rightarrow \Lambda^{2} \mathbf{M}$ induces a symmetric endomorphism of $\Lambda^{2} \mathbf{M} \otimes \Lambda^{1} \mathbf{M}$, which maps the two above spaces isomorphically into each other. This implies that $C^{+}$and $C^{-}$satisfy (10) and (11) (note that these two relations are equivalent in their case) and are, therefore, $S O(4, \mathbb{C})$-irreducible.

The Cotton-York tensor is related to the Weyl tensor of $\mathbf{M}$ by the formula [5]

$$
\delta W=C,
$$

where $\delta: \Gamma\left(\Lambda^{2} \mathbf{M} \otimes \Lambda^{2} \mathbf{M}\right) \rightarrow \Gamma\left(\Lambda^{2} \mathbf{M} \otimes \Lambda^{1} \mathbf{M}\right)$ is induced by the codifferential on the second factor, and by the Levi-Civita connection $\nabla$ on the first. Then, $C^{+}$has to be the component of $\delta W$ in $\Lambda^{+} \mathbf{M} \otimes \Lambda^{1} \mathbf{M}$, and we know that the restriction of $W^{-}$to $\Lambda^{+} \mathbf{M} \otimes \Lambda^{2} \mathbf{M}$ is identically zero. This means that

$$
\begin{aligned}
& \delta W^{+}=C^{+}, \text {and also } \\
& \delta W^{-}=C^{-} .
\end{aligned}
$$

Hence, as $\mathbf{M}$ is self-dual, $C^{-}$vanishes identically.

We can prove now that the Thomas tensor of a $\beta$-surface $\beta$ is identically zero. First we show that

$$
K(Y) X=\left.\operatorname{tr}\right|_{T \beta} R(Y, \cdot) X=h(X, Y), \forall X, Y \in T \beta .
$$

We recall from [5] that the suspension $h \wedge \mathbf{I}$ of $h$ by the identity, viewed as an endomorphism of $\Lambda^{2} \mathbf{M}$, is defined by

$$
(h \wedge \mathbf{I})(X, Y):=h(X) \wedge Y-h(Y) \wedge X, X, Y \in T \mathbf{M}
$$

where $h$ is identified with a symmetric endomorphism of $T \mathbf{M}$.

We have then the following decomposition of the Riemannian curvature [5]:

$$
R=h \wedge \mathbf{I}+W^{+}+W^{-} .
$$

Of course, if $\mathbf{M}$ is self-dual, then $W^{-}=0$ and $W^{+}(X, Y)=0$ if $X, Y \in T \beta$ (in fact, the elements in $\Lambda^{2} F^{\beta}$, for any $\beta$-plane $F^{\beta} \subset T_{x} \mathbf{M}$, correspond to the isotropic vectors in $\left.\Lambda^{-} \mathbf{M}\right)$, because $\left.W^{+}\right|_{\Lambda^{-} M}=0$. Then, if we choose the basis $\{X, Y\}$ in 
$T \beta$, we get

$$
\begin{aligned}
K(Y) X & =\left.\operatorname{tr}\right|_{T \beta}(h \wedge \mathbf{I})(Y, \cdot) X \\
& =\text { the component along } X \text { of }(h \wedge \mathbf{I})(Y, X) X \\
& =h(Y, X),
\end{aligned}
$$

which proves (15). The Thomas tensor of the projective structure of $\beta$ has the following expression (see (7)):

$$
T(X, Y, Z)=-3\left(\nabla_{Z} h\right)(Y, X)+3\left(\nabla_{Y} h\right)(Z, X)=3 C(Y, Z)(X), \forall X, Y, Z \in T \beta,
$$

and, as $C^{+}(\cdot, \cdot)(X)$ vanishes on the anti-self-dual 2 -form $Y \wedge Z$, we conclude that

$$
T(X, Y, Z)=C^{-}(Y, Z)(X)=0 .
$$

As the flatness of the projective structure on a 2-dimensional manifold is equivalent to the vanishing of its Thomas tensor [20, we get

Corollary 2. The projective structure of the $\beta$-surfaces of a self-dual complex manifold $\mathbf{M}$ is flat.

From the classification of projectively flat compact complex surfaces ( 9 , see also 7]), we then get a classification of compact $\beta$-surfaces in $\mathbf{M}$ :

Theorem 4. A compact $\beta$-surface of a self-dual complex 4-manifold belongs (up to finite covering) to one of the following classes:

(1) $\mathbb{C P}^{2}$;

(2) a compact quotient of the complex-hyperbolic plane $\mathbf{H}_{\mathbb{C}}^{2} / \Gamma$;

(3) a compact complex surface admitting a (flat) affine structure:

(i) a Kodaira surface;

(ii) a properly elliptic surface with $b_{1}$ odd;

(iii) an affine Hopf surface;

(iv) an Inoue surface;

(v) a complex torus.

See [7, [9, 11] for details.

\section{EXAMPLES}

7.1. The flat case. The first example is the "flat" case: $Z=\mathbb{C P}^{3}=\mathbb{P}\left(\mathbb{C}^{4}\right)$, with its canonical projective structure and its space of projective lines $\mathbf{M}=\operatorname{Gr}\left(2, \mathbb{C}^{4}\right) .(Z$ is equally the twistor space of the Riemannian round 4-sphere, which is, therefore, a real part of $\operatorname{Gr}\left(2, \mathbb{C}^{4}\right)$.) If $\beta \in Z$, then the $\beta$-surface associated to it is the set $\left\{x \in \operatorname{Gr}\left(2, \mathbb{C}^{4}\right) \mid \beta \subset x \subset \mathbb{C}^{4}\right\}$. In this flat case, we can equally define the $\alpha$-twistor space $Z^{*}$, which is the dual projective 3 -space $\left(\mathbb{C P}^{3}\right)^{*}:=\mathbb{P}\left(\left(\mathbb{C}^{4}\right)^{*}\right)=\operatorname{Gr}\left(3, \mathbb{C}^{4}\right)$, and an $\alpha$-surface $\alpha \in Z^{*}$ is the set $\left\{x \in \operatorname{Gr}\left(2, \mathbb{C}^{4}\right) \mid x \subset \alpha \subset \mathbb{C}^{4}\right\} \subset \mathbf{M}$. A null-geodesic $\gamma$ is then determined by a pair of incident isotropic surfaces $\alpha$ and $\beta$ such that $\alpha \cap \beta=\gamma$, where $\alpha$ is an $\alpha$-surface and $\beta$ is a $\beta$-surface; incident means (see above) that $\beta$, seen as a line in $\mathbb{C}^{4}$, is included in $\alpha$, seen as a 3-plane in $\mathbb{C}^{4} . \gamma$ is then the following set of points in $\mathbf{M}$ :

$$
\gamma=\left\{x \in \operatorname{Gr}\left(2, \mathbb{C}^{4}\right) \mid \beta \subset x \subset \alpha\right\} .
$$




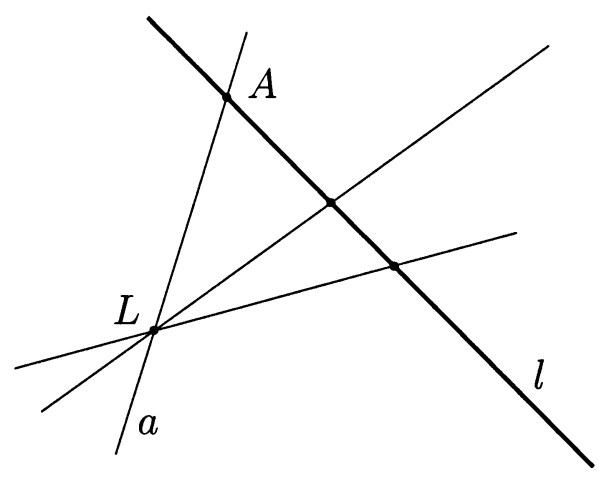

FIGURE 4.

$\alpha$-surfaces and $\beta$-surfaces are diffeomorphic to $\mathbb{C P}^{2}$, null-geodesics to $\mathbb{C P}^{1}$, and the ambitwistor space $B$ is the "partial flag" manifold

$$
B=\left\{(\alpha, \beta) \in\left(\mathbb{C P}^{3}\right)^{*} \times \mathbb{C P}^{3} \mid \beta \subset \alpha\right\} .
$$

The flag manifold, of dimension 7 , is isomorphic to the total space $\mathbb{P}(C)$ of the projective cone bundle over $\mathbf{M}$.

7.2. $\mathbb{C P}^{2}$. Another example is when $Z$ is the twistor space of the real Riemannian manifold $\mathbb{C P}^{2}$, with the Fubini-Study metric. Then $Z$ is the manifold of flags in $E=\mathbb{C}^{3}, \mathcal{F}:=\left\{(L, l) \in \mathbb{P}(E) \times \mathbb{P}(E)^{*} \mid L \subset l\right\}\left(\mathbb{P}(E)\right.$, resp. $\mathbb{P}(E)^{*}$ are viewed as the space of lines, resp. 2-planes, in $E)$ [1. A projective line $Z_{x}$ in $Z$ is a set

$$
Z_{x}=\left\{(L, l) \in \mathcal{F} \mid L \subset a^{x}, A^{x} \subset l\right\}
$$

(see Figure 4), where $\left(A^{x}, a^{x}\right)$ belongs to $\mathbb{P}(E) \times \mathbb{P}(E)^{*} \backslash \mathcal{F}$, which is, therefore, the space $\mathbf{M}$ of such lines, and a conformal self-dual 4 -manifold. It can be naturally compactified within the space of analytic cycles of $Z$ to $\overline{\mathbf{M}}=\mathbb{P}(E) \times \mathbb{P}(E)^{*}$, which is obviously a smooth manifold, but it carries no global conformal structure, as its canonical bundle has no square root. This means that the conformal structure on $\overline{\mathbf{M}}$ is smooth on $\mathbf{M}$, and singular on $\mathcal{F}=\overline{\mathbf{M}} \backslash \mathbf{M}$. The cycles of $Z$ corresponding to a point $\bar{x}=(A, a)$ in this subset are pairs of complex projective lines in $Z$ :

$$
Z_{\bar{x}}=\{(A, l) \in Z=\mathcal{F}\} \cup\{(L, a) \in Z=\mathcal{F}\} .
$$

A $\beta$-surface in $\mathbf{M}$, corresponding to a point $\beta=(L, l) \in Z$, is the set

$$
\beta=\left\{(A, a) \in \mathbb{P}(E) \times \mathbb{P}(E)^{*} \mid A \subset l, L \subset a, A \neq L, a \neq l\right\},
$$

and can be naturally compactified to

$$
\bar{\beta}=\left\{\left(A, l^{\beta}\right) \in \mathcal{F}\right\} \times\left\{\left(L^{\beta}, a\right) \in \mathcal{F}\right\} \simeq \mathbb{C P}^{1} \times \mathbb{C P}^{1} .
$$

7.3. The tangent space to $\mathcal{F}$. In order to describe the null-geodesics of $\mathbf{M}$ as 2-planes in $Z$, we first study the tangent space of $Z=\mathcal{F}$ at $\beta=(L, l)$.

A vector in $T_{(L, l)} \mathcal{F}$ is a pair of vectors $(V, v)$, with $V \in T_{L} \mathbb{P}(E)$ and $v \in T_{l} \mathbb{P}(E)^{*}$, which satisfy a linear condition $\left(\right.$ as $\left.\mathcal{F} \subset \mathbb{P}(E) \times \mathbb{P}(E)^{*}\right)$. Actually, there is a duality 


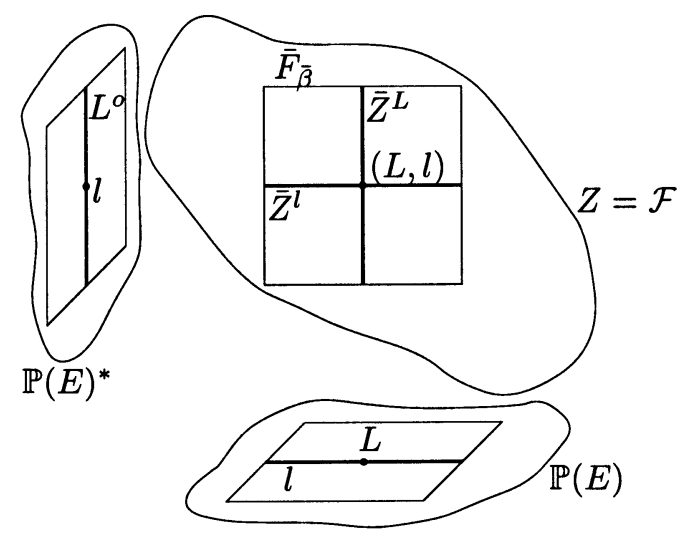

FiguRE 5 .

between $\mathbb{P}(E)^{*}$, the Grassmannian of 2-planes in $E$, and $\mathbb{P}\left(E^{*}\right)$, the projective space of $E^{*}:=\operatorname{Hom}(E, \mathbb{C})$, and an analogous one between $\mathbb{P}(E)$ and $\mathbb{P}\left(E^{*}\right)^{*}$ :

$$
\begin{aligned}
& \mathbb{P}(E)^{*} \ni l \stackrel{\simeq}{\longmapsto} l^{o} \in \mathbb{P}\left(E^{*}\right), \\
& \mathbb{P}(E) \ni L \stackrel{\cong}{\longmapsto} L^{o} \in \mathbb{P}\left(E^{*}\right)^{*} .
\end{aligned}
$$

Then the flag manifold $\mathcal{F}$ is defined, as a submanifold of $\mathbb{P}(E) \times \mathbb{P}(E)^{*}$, by the equation

$$
y(Y)=0, \forall y \in l^{o}, \forall Y \in L
$$

The vector $V \in T_{L} \mathbb{P}(E)$ is an element in $\operatorname{Hom}(L, E / L)$. By duality, $v \simeq v^{o} \in$ $\operatorname{Hom}\left(l^{o}, E^{*} / l^{o}\right)$. Then the vector $(V, v) \in T_{(L, l)} \mathbb{P}(E) \times \mathbb{P}(E)^{*}$ lies in $\mathcal{F}$ iff

$$
v^{o}\left(y^{o}\right)(Y)+y^{o}(V(Y))=0, \forall Y \in L, \forall y^{o} \in l^{o},
$$

or, equivalently,

$$
\left.v\right|_{L}=\pi_{l} \circ V
$$

where $\pi_{l}: E / L \rightarrow E / l$ is the projection (as $L \subset l$ ).

The geometry of $\mathcal{F}$, as a subset of $\mathbb{P}(E) \times \mathbb{P}(E)^{*}$, can be described as in Figure 5 .

7.4. The 2-planes in $\mathcal{F}$. Let us consider now a 2-plane $F$ in $T_{(L, l)} \mathcal{F}$, and the cycles (corresponding to points in $\overline{\mathbf{M}}$ ) tangent to it. We have three cases:

1. $F=\bar{F}_{\beta}$ is the "degenerate" 2-plane tangent to the 2 special curves $\bar{Z}_{L}, \bar{Z}_{l}$ whose union is the special cycle $\left.\bar{F}_{(} L, l\right)$ corresponding to $(L, l) \in \overline{\mathbf{M}} \backslash \mathbf{M}$. There are no projective lines $Z_{x}, x \in \mathbf{M}$, tangent to it; only the special cycles $\bar{Z}_{(L, a)}, L \subset a$, and $\bar{Z}_{(A, l)}, A \subset l$, are tangent to $\bar{F}_{(L, l)}$, actually only to the two privileged directions of $\bar{Z}_{L}$, resp. $\bar{Z}_{l}$.

Remark. The special curves $\bar{Z}_{L}, \bar{Z}_{l}$ have trivial normal bundle, being fibers of the projections from $\mathcal{F}$ to $\mathbb{P}(E)$, resp. $\mathbb{P}(E)^{*}$, so these special curves form two complete families of analytic cycles in $\mathcal{F}$, isomorphic to $\mathbb{P}(E)$, resp. $\mathbb{P}(E)^{*}$. Two such curves are incident iff they are of different types $\left(\bar{Z}_{L}\right.$ is of type $E, \bar{Z}_{l}$ is of type $\left.E^{*}\right)$, so they can only form "polygons" with an even number of edges. But there are no quadrilaterals, as one can easily check, using the fact that $\bar{Z}_{L}$ and $\bar{Z}_{l}$ are incident 


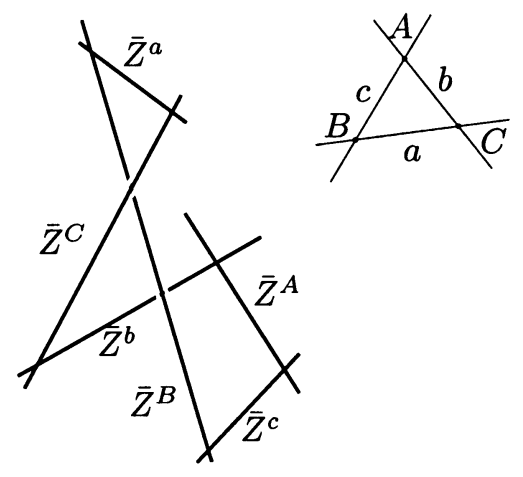

FiguRE 6.

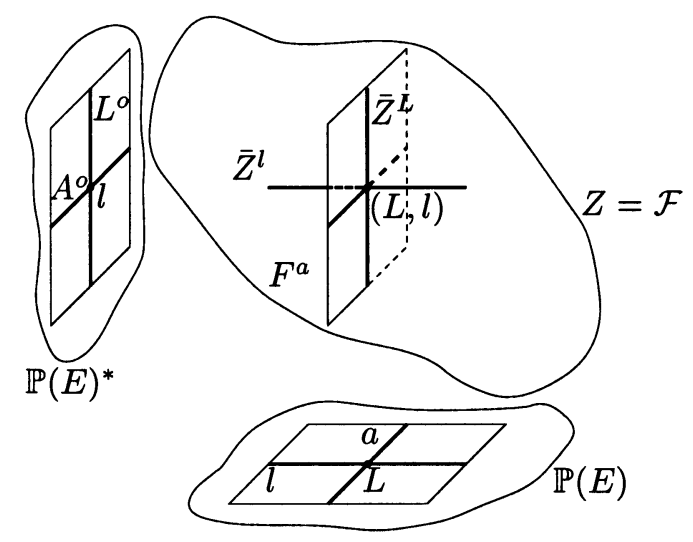

FiguRE 7.

iff $L \subset l$, thus iff $l$ is a line in $\mathbb{P}(E)$ containing $L$. On the other hand, there are hexagons, corresponding to the 3 vertices and 3 sides of a triangle in $\mathbb{P}(E) \simeq \mathbb{C P}^{2}$ (see Figure 6).

The above hexagon is not "flat", i.e. there is no canonical submanifold of $\mathcal{F}$ containing it. This, and the fact that there are no quadrilaterals made of $\bar{Z}$-type curves, is just a consequence of the fact that the distribution $\bar{F}$ on $Z=\mathcal{F}$ is not integrable; in fact it is the holomorphic contact structure induced by the FubiniStudy Einstein metric on $\mathbb{C P}^{2}$ ([3]; see also Section 7.6$)$.

2. $F=F^{a}$, for $a \supset L, a \neq l$. This is a 2-plane that is tangent to only one of the special curves $\bar{Z}_{L}$. The projective lines tangent to $F^{a}$ at $\beta=(L, l)$ are $Z_{(A, a)}$, $\forall A \subset l, A \neq L$; hence the corresponding null-geodesic is

$$
\gamma^{a}=\left\{(A, a) \in \mathbb{P}(E) \times \mathbb{P}(E)^{*} \mid A \subset l, A \neq l\right\},
$$

thus it is diffeomorphic to $\mathbb{C}$, and its closure is

$$
\bar{\gamma}^{a}=\left\{(A, a) \in \mathbb{P}(E) \times \mathbb{P}(E)^{*} \mid A \subset l\right\} \simeq \mathbb{C P}^{1}
$$

(see Figure 7). 


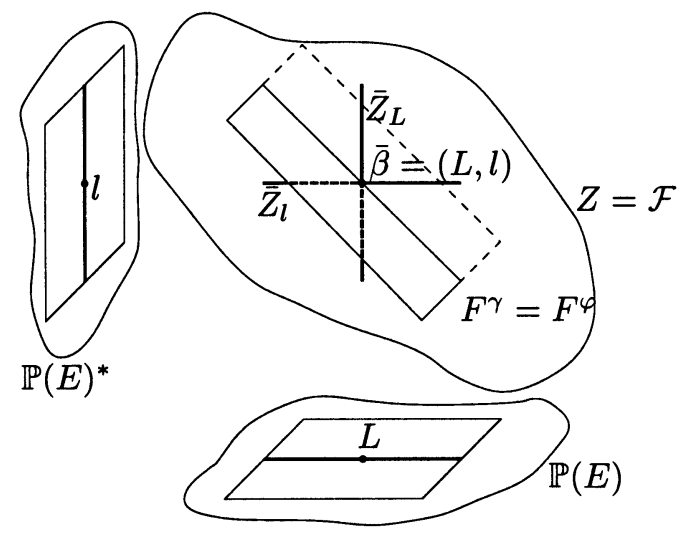

FIGURE 8.

Remark. The "limit" curve is $\bar{Z}_{(L, a)}$, so it is non-singular at $(L, l)$. Actually, the points of $Z_{(A, a)}$ close to $(L, l)$ converge, when $A \rightarrow L$, to some points in $\bar{Z}_{L}$, which is tangent to $F^{a}$. We can then apply the same method as in Theorem 2 to conclude that the integral $\alpha$-cone associated to $F^{a}$ is a smooth manifold around $(L, l)$; thus, from Theorem 1, the Weyl tensor $W^{+}$of $\mathbf{M}$ vanishes on the $\beta$-planes generated along $\gamma^{a}$ by its own direction. We will see that the vanishing of $W^{+}$ on these $\alpha$-planes leads to the existence of some $\alpha$-surfaces, see below. Of course, the deformation argument in Theorem 2 does not hold in the present case, as the normal bundle of $\bar{Z}_{L}$ is trivial, thus different from that of the rest of the rational curves $Z_{(A, a)}$ (as we will see below, generic 2-planes through $(L, l)$ do not admit projective lines tangent to all their directions).

$\mathbf{2}^{\prime}$. We have a similar situation for planes $F=F^{A}-A \subset l, A \neq L$, tangent to the other special curve $\bar{Z}_{l}$.

3. This is the generic case: $F=F^{\varphi}$, where $\varphi: \mathbb{P}(l) \rightarrow \mathbb{P}\left(L^{o}\right)$ is a projective diffeomorphism such that $\varphi(L)=l^{o}$. Indeed, the tangent spaces $T_{L} \mathbb{P}(E)$ and $T_{l} \mathbb{P}(E)^{*}$ are isomorphic to $\operatorname{Hom}\left(L^{o}, E^{*} / L^{o}\right)$, resp. to $\operatorname{Hom}(l, E / l)$, and a generic 2-plane $F$ in $T_{(L, l)} \mathcal{F}$ is the graph of a linear isomorphism $\phi: T_{L} \mathbb{P}(E) \rightarrow T_{l} \mathbb{P}(E)^{*}$ satisfying a linear condition (18) or (19). Actually, the graph is determined by the projective application $\varphi$ induced by $\phi$ from $\mathbb{P}\left(T_{L} \mathbb{P}(E)\right) \simeq \mathbb{P}\left(L^{o}\right)$ to $\mathbb{P}\left(T_{l} \mathbb{P}(E)^{*}\right) \simeq$ $\mathbb{P}(l)$ (see Figure 8).

The condition $\varphi(L)=l^{\circ}$ is implied by (19). The null-geodesic associated to the 2-plane $F^{\varphi}$ is

$$
\gamma^{\varphi}=\left\{(A, a) \in \mathbb{P}(E) \times \mathbb{P}(E)^{*} \backslash \mathcal{F} \mid A \subset l, a^{o} \subset L^{o} a^{o}=\varphi(A)\right\},
$$

and its closure in $\overline{\mathbf{M}}$ is

$$
\bar{\gamma}^{\varphi}=\left\{(A, a) \in \mathbb{P}(E) \times \mathbb{P}(E)^{*} \mid A \subset l, a^{o} \subset L^{o}\right\} .
$$

Hence the "limit" point is $(L, l) \in \overline{\mathbf{M}}$, corresponding to the special cycle $\bar{Z}_{(L, l)}$, none of whose components is tangent to $F^{\varphi}$. The integral $\alpha$-cone associated to $F^{\varphi}$ looks like what is shown in Figure 9.

7.5. The null-geodesics of the complexification of $\mathbb{C P}^{2}$. The application $\varphi$ has the following interpretation in terms of projective geometry on $\mathbb{C P}^{2}=\mathbb{P}(E)$ : a 


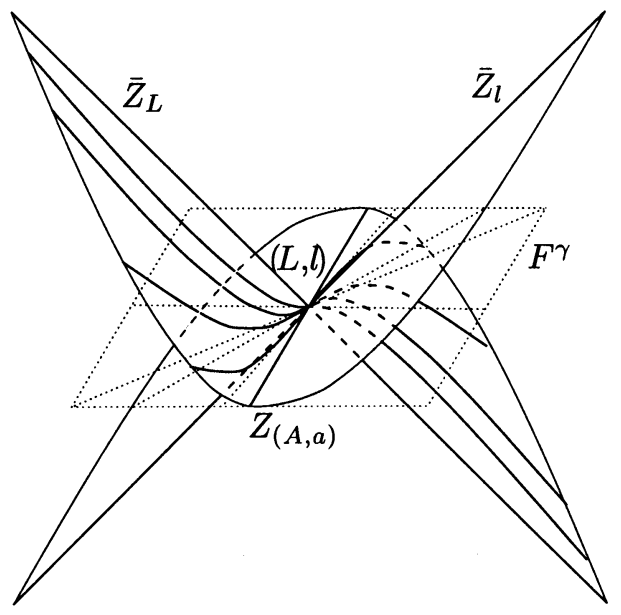

FIGURE 9.

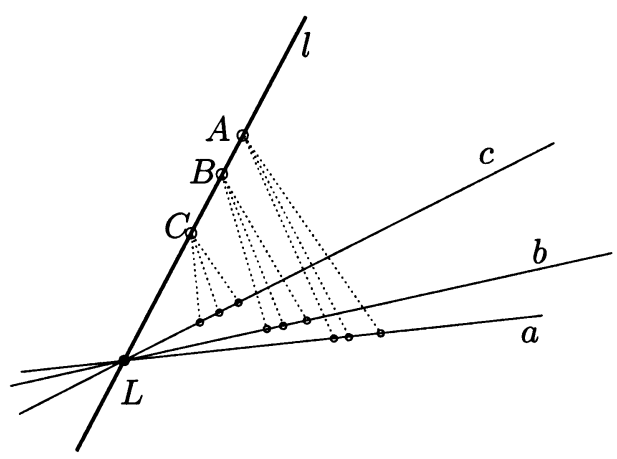

FiguRe 10.

direction $\mathbb{C} v$ in $T_{l} \mathbb{P}(E)^{*}$ is identified with the point $\operatorname{ker} v \equiv A \in l / L \subset \mathbb{P}(E)$ and a direction $\mathbb{C} V \subset T_{L} \mathbb{P}(E)$ is identified with a direction (thus a projective line $a$ ) through $L \in \mathbb{P}(E) . \varphi$ is, thus, a homography that associates to $A \in l$ (we identify $l$ with the projective line $l / L \subset \mathbb{P}(E))$ the line $a \ni L$. As $\varphi(L)=l$, we have, then, that three points $(A, a),(B, b),(C, c) \in \beta^{(L, l)}$ belong to the same null-geodesic iff

$$
(A, B: C, L)=(a, b: c, l) \text {, }
$$

i.e. the cross-ratio of the points $A, B, C, L \in l$ equals the cross-ratio of the lines $a, b, c, l$ through $L$ (the dotted lines, together with their intersections with the lines $a, b, c$, correspond to the points in the integral $\alpha$-cone; see Figure 10).

We can now describe the null-geodesics passing through a point $(A, a) \in \mathbf{M}$ and contained in a $\beta$-surface $\left.\beta^{(} L, l\right)$ whose closure $\bar{\beta}$ is isomorphic to $\mathbb{C P}^{1} \times \mathbb{C P}^{1}$ : they coincide with the rational curves in $\bar{\beta}$ containing $(A, a)$; except the "horizontal" $\left(\bar{\gamma}^{A}\right)$ and "vertical" $\left(\bar{\gamma}^{a}\right)$ ones, all these curves contain $(L, l)$; see Figure 11.

We remark that, in the usual affine coordinates on

$$
\beta \simeq\left(\mathbb{C P}^{1} \backslash\{L\}\right) \times\left(\mathbb{C P}^{1} \backslash\{l\}\right) \simeq \mathbb{C}^{2},
$$




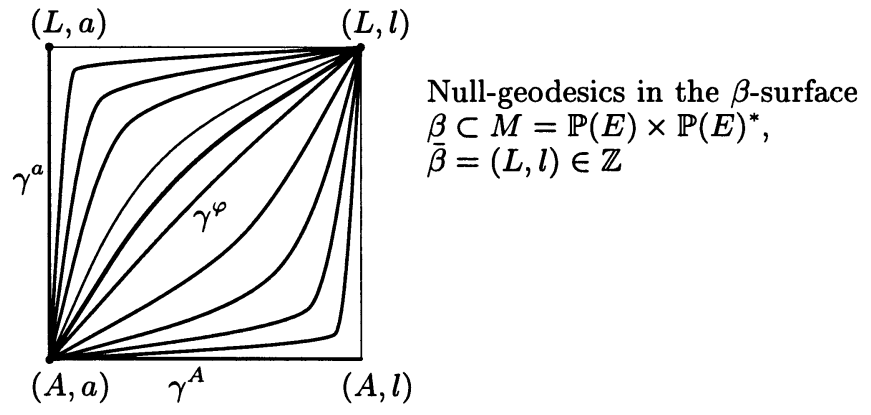

Figure 11.

these null-geodesics are the affine lines containing $(A, a)$; thus the projective structure on $\beta$ is (locally) isomorphic to a flat affine structure. We have seen, in Section 6)(Corollary 2), that this is true for all $\beta$-surfaces of a self-dual manifold.

7.6. The conformal structure of the complexification of $\mathbb{C P}^{2}$. Now let us study the conformal structure of $\mathbf{M}=\mathbb{P}(E) \times \mathbb{P}(E)^{*} \backslash \mathcal{F}$ directly; actually $\mathbf{M}$ has a complex metric $g$. Let $(A, a) \in \mathbf{M}$; then $A$ is transverse to $a$, and so we have the isomorphisms $E / a \simeq A$ and $E / A \simeq a$. Then, a vector $(V, v) \in T_{(A, a)} \mathbf{M}$ is identified with a pair of homomorphisms $V: A \rightarrow a$ and $v: a \rightarrow A$, and the metric $g$ is given by

$$
g((V, v),(W, w)):=\operatorname{tr}(v \circ W+w \circ V), \quad \forall(V, v),(W, w) \in T_{(A, a)} \mathbf{M} .
$$

Remark (The real part). Let $h$ be an Hermitian metric on $E$. Then we have a real-analytic embedding of $\mathbf{M}_{0} \simeq \mathbb{P}(E)$ into $\mathbf{M}$, given by

$$
\mathbb{P}(E) \ni A \longmapsto\left(A, A^{\perp}\right) \in \mathbb{P}(E) \times \mathbb{P}(E)^{*} \backslash \mathcal{F} .
$$

A vector $(V, v) \in T_{\left(A, A^{\perp}\right)} \mathbf{M}$ is tangent to $\mathbf{M}_{0}$ iff

$$
h(x, v(y))+h(V(x), y)=0, \forall x \in A, \forall y \in A^{\perp} .
$$

Then one easily checks that

$$
g((V, v),(W, w))=-2 h(V, W), \forall(V, v),(W, w) \in T_{\left(A, A^{\perp}\right)} \mathbf{M}_{0} .
$$

Hence, up to a constant, the restriction of $g$ to $\mathbf{M}_{0} \simeq \mathbb{C P}^{2}$ is the Fubini-Study metric of $\mathbb{C P}^{2} \simeq S^{5} / S^{1}$.

An isotropic vector in $\mathbf{M}$ is $(V, v) \in T_{(A, a)} \mathbf{M}$, with $v \circ V=0$, viewed as an endomorphism of $A$ (see above), or, equivalently, with

$$
\operatorname{dim}(A+V(A) \cap \operatorname{ker} v)>0 .
$$

Let us see which is the limit of the isotropic cone in the points of $\mathcal{F}$ : from the relation above, it follows that the isotropic cone at a point $x \in \mathcal{F}$ is

$$
C_{x}=\left\{(0, v) \in T_{x} \mathcal{F}\right\} \cup\left\{(V, 0) \in T_{x} \mathcal{F}\right\},
$$

so the conformal structure of $\mathbf{M}$ is singular at the "infinity" $\mathcal{F}$. 
Remark. The situation $\mathcal{F} \subset \mathbb{P}(E) \times \mathbb{P}(E)^{*}$ is very similar to the one treated in 2]; see also [12]: $\mathbb{P}(E) \times \mathbb{P}(E)^{*}$ has an Einstein self-dual metric $g$, singular at the "infinity", and this Einstein structure yields a contact structure on the twistor space $Z=\mathcal{F}$; the field of 2-planes determined by this contact structure corresponds to the "infinity" $\mathcal{F} \subset \mathbb{P}(E) \times \mathbb{P}(E)^{*}$. But these planes do not admit tangent rational curves with normal bundle $\mathcal{O}(1) \oplus \mathcal{O}(1)$ : the conformal structure does not extend to the "infinity" (which is, therefore, not a conformal infinity).

7.7. $\alpha$-planes and $\beta$-planes. We consider the isotropic planes in $T_{(A, a)} \mathbf{M}(A \not \subset$ $a)$ : For a fixed isotropic direction, represented by a generic vector $(V, v) \in T_{(A, a)} \mathbf{M}$, the line $\operatorname{ker} v \subset a$ and the plane $V(A)+A \supset A$ are fixed. The linear space of all vectors $(W, v) \in T_{(A, a)} \mathbf{M}$ satisfying

$$
W(A) \subset A+V(A),\left.\quad w\right|_{\operatorname{ker} v}=0,
$$

is isotropic and orthogonal to $(V, v)$ : they form a $\beta$-plane. The $\alpha$-plane $F^{\alpha}$ containing $(V, v)$ corresponds to the isotropic vectors $(W, w)$ orthogonal to $(V, v)$ with $\operatorname{ker} w \neq \operatorname{ker} v$. As a plane transverse to all the $\beta$-planes (whose projection onto $T_{A} \mathbb{P}(E)$ or $T_{a} \mathbb{P}(E)^{*}$ is never injective), $F^{\alpha}$ is determined by a linear isomorphism $\varphi: T_{A} \mathbb{P}(E) \rightarrow T_{a} \mathbb{P}(E)^{*}$, whose graph in $T_{(A, a)} \mathbb{P}(E) \times \mathbb{P}(E)^{*}$ is $F^{\alpha} ; \varphi$ induces the application $\mathbb{P} \varphi: \mathbb{P}(a) \rightarrow \mathbb{P}(E / A)$ between the projective spaces of $T_{A} \mathbb{P}(E)$, resp. $T_{a} \mathbb{P}(E)^{*}$. The plane $F^{\alpha}=F^{\varphi}$, the graph of $\varphi$, is isotropic iff $V \subset \mathbb{P} \varphi(V)$, $\forall V \in \mathbb{P}(a)$, i.e. $\mathbb{P} \varphi$ is the homography that sends a point $X$ in $a$ into the projective line through $A$ and $X$. We can extend $\varphi$ to a projective isomorphism $\varphi^{\prime}: \mathbb{P}\left(\mathbb{C} \oplus T_{A} \mathbb{P}(E)\right) \rightarrow \mathbb{P}\left(\mathbb{C} \oplus T_{a} \mathbb{P}(E)^{*}\right)$ : for example, $\mathbb{P}\left(\mathbb{C} \oplus T_{A} \mathbb{P}(E)\right)$ contains $T_{A} \mathbb{P}(E)$ as an affine open set. Then $\varphi^{\prime}$ is defined as follows:

$$
\begin{aligned}
\left.\varphi^{\prime}\right|_{T_{A} \mathbb{P}(E)} & :=\varphi, \\
\left.\varphi^{\prime}\right|_{\mathbb{P}\left(T_{A} \mathbb{P}(E)\right)} & :=\mathbb{P} \varphi .
\end{aligned}
$$

Actually $\mathbb{P}\left(\mathbb{C} \oplus T_{A} \mathbb{P}(E)\right) \simeq \mathbb{P}(E)$ and $\mathbb{P}\left(\mathbb{C} \oplus T_{a} \mathbb{P}(E)^{*}\right) \simeq \mathbb{P}(E)^{*}$. We then have

Proposition 7. A generic $\alpha$-plane $F^{\alpha}=F^{\varphi}$ in $T_{(A, a)} \mathbf{M}$ is the graph of a linear isomorphism $\varphi: T_{A} \mathbb{P}(E) \rightarrow T_{a} \mathbb{P}(E)^{*}$, which is determined by a projective isomorphism

$$
\varphi^{\prime}: \mathbb{P}(E) \rightarrow \mathbb{P}(E)^{*}
$$

such that $\varphi^{\prime}(A)=a$ and $\varphi^{\prime}(l)=l \cap a$, for all $l \supset A$.

7.8. Exponentials of $\alpha$-planes. The exponential $\exp \left(F^{\varphi}\right)$ has an interpretation in terms of projective geometry. Each direction $\mathbb{C}(V, v) \subset F^{\varphi}$ is determined by the point ker $v$ in $a \subset \mathbb{P}(E)$ and the line through $A$ and ker $v$, and a homography $\phi^{(V, v)}$ from the points $B$ of the projective line $A+\operatorname{ker} v$ to the space of lines $b$ through ker $v$ (see Figure 12 and the convention below). As this homography is the restriction of $\varphi^{\prime}$ to the appropriate spaces, it follows that it is related to the homography $\phi^{(W, w)}$, where $\mathbb{C}(W, w)$ is another direction in $F^{\varphi}$ : the points $D:=b \cap c, P:=a \cap(B+C)$ and $A$ are collinear (see Figure 12).

Of course, this implies that $P$ determines a homography $\psi^{P}$ between the lines $A+\operatorname{ker} v$ and $A+\operatorname{ker} w$, such that $\psi^{P}(A)=A$ and $\psi^{P}(\operatorname{ker} v)=\operatorname{ker} w$. Then, for any other points $B^{\prime} \in(A+\operatorname{ker} v), C^{\prime}=\psi^{P}(B) \in(A+\operatorname{ker} w)$, the lines $b^{\prime}=$ $\phi^{(V, v)}\left(B^{\prime}\right), c^{\prime}=\phi^{(W, w)}\left(C^{\prime}\right)$ intersect on the line $(A+P)$ (see the right-hand side of Figure 12). 

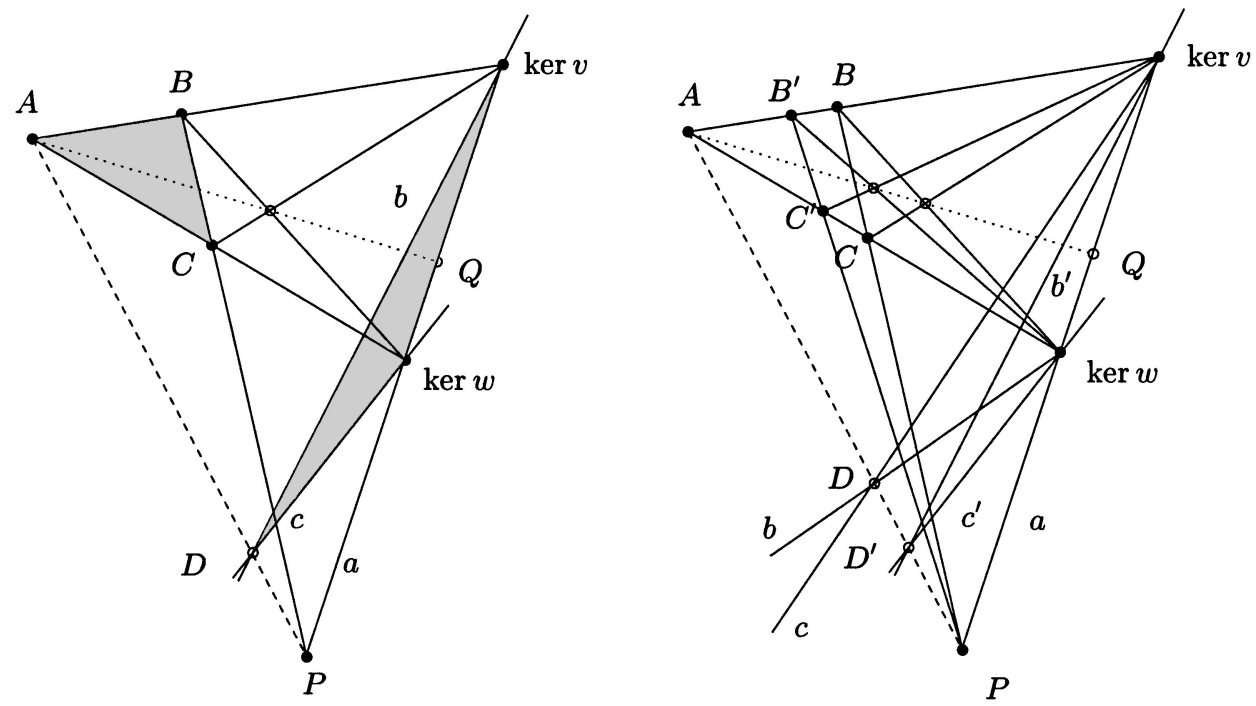

FigURE 12.

Convention. In the framework of plane projective geometry, we identify a point in $\mathbb{P}(E)^{*}$ with a line in $\mathbb{P}(E)$ (we denote, for example, ker $v \in a$ ). The lines determined by the distinct points $B$ and $C$ will be denoted by $(B+C)$ (thus $B, C \in(B+C)$ ).

The null-geodesic tangent to $(V, v)$ at $(A, a)$ is the set $\{(B, b) \mid B \in(A+\operatorname{ker} v), b=$ $\left.\phi^{(V, v)}(B)\right\}$, and the null-geodesic tangent to $(W, w)$ is the analogous set of the pairs $(C, c)$. Thus

$$
\begin{gathered}
\exp _{(A, a)}\left(F^{\varphi}\right)=\exp _{(A, a)}\left(F^{\alpha}\right)=\{(C, c) \mid C \in \mathbb{P}(E), C \neq A, \\
\left.c=((C+A) \cap a)+\left((A+P) \cap b^{C}\right)\right\} \cup\{(A, a)\},
\end{gathered}
$$

where $B^{C}:=(A+\operatorname{ker} v) \cap(P+C)$, and $b^{C}:=\phi^{(V, v)}\left(B^{C}\right)$, as in Figure 12 (where $\left.B=B^{C}, b=b^{C}\right)$. This gives the exponential of the $\alpha$-plane determined by the isotropic vector $(V, v)$. We remark that the point $(A, a)$ has a privileged position in $\exp _{(A, a)}\left(F^{\alpha}\right):(a \cap b) \in(A+B) \forall(B, b) \in \exp _{(A, a)}\left(F^{\alpha}\right)$; on the other hand, $(b \cap c) \notin(B+C)$ in general (see Figure 12), which means that the points $(B, b)$ and $(C, c)$ are not null-separated (i.e. they do not belong to the same null-geodesic). That means that $\exp _{(A, a)}\left(F^{\alpha}\right)$ is not totally isotropic; thus there is no $\alpha$-surface tangent to a generic $\alpha$-plane - not surprising, as the corresponding $\alpha$-cone is not flat (see Section 7.4).

But there are $\alpha$-surfaces tangent to the two $\alpha$-planes $\left\{(V, 0) \mid V \in T_{A} \mathbb{P}(E)\right\}$ and $\left\{(0, v) \mid v \in T_{a} \mathbb{P}(E)^{*}\right\}$ : the "slices" $\{A\} \times \mathbb{P}(E)^{*}$ and $\mathbb{P}(E) \times\{a\}$. (It is easy to see that these planes are isotropic, and that they are not $\beta$-planes, as these project on lines in $T_{A} \mathbb{P}(E)$, resp. $T_{a} \mathbb{P}(E)^{*}$.)

Thus $\mathbf{M}=\mathbb{P}(E) \times \mathbb{P}(E)^{*} \backslash \mathcal{F}$ is a conformal self-dual manifold, not anti-self-dual, that admits $\alpha$-surfaces passing through any point. 


\section{ACKNOWLEDGEMENTS}

The author is deeply indebted to Paul Gauduchon, for his care in reading the manuscript and for his constant help during the research and redaction.

\section{REFERENCES}

[1] M.F. Atiyah, N.J. Hitchin, I.M. Singer, Self-duality in four-dimensional Riemannian geometry, Proceedings of the Royal Society of London, Series A 362 (1978), 425-461. MR 80d:53023

[2] F.A. Belgun, Null-geodesics on complex conformal mmanifolds and the LeBrun correspondence, J. Reine Angew Math. 536 (2001), 43-63. MR 2002g:53080

[3] A.L. Besse, Einstein manifolds, Springer Verlag (1987). MR 88f:53087

[4] F. Campana, On twistor spaces of the class $\mathcal{C}$, J. Diff. Geom. 33 (1991), 541-549. MR 92g:32059

[5] P. Gauduchon, Connexion canonique et structures de Weyl en géométrie conforme, Preprint, juin 1990.

[6] N.J. Hitchin, Complex manifolds and Einstein's equations, in Twistor Geometry and Nonlinear Systems, Lecture Notes in Mathematics 970 (1982) Springer-Verlag, 73-99. MR 84i:32041

[7] B. Klingler, Structures affines et projectives sur les surfaces complexes, Ann. Inst. Fourier 48 (1998), 441-477. MR 99c:32038

[8] S. Kobayashi, K. Nomizu, Foundations of Differential Geometry I, Interscience, John Wiley (1963). MR 27:2945

[9] S. Kobayashi, T. Ochiai, Holomorphic projective structures on compact complex surfaces, Math. Annalen, 249 (1980), 75-94. MR 81g:32021

[10] K. Kodaira, A theorem of completeness of characteristic systems for analytic families of compact submanifols of complex manifolds, Ann. Math., II Ser.,75 (1962), 146-162. MR 24:A3665b

[11] K. Kodaira, On compact complex analytic spaces I, Ann. Math. 71 (1960), 111-152; II, ibid. 77 (1963), 563-626; III, ibid. 78 (1963), 1-40. MR 24:A2396; MR 32:1730

[12] C.R. LeBrun, $\mathcal{H}$-Space with a cosmological constant, Proceedings of the Royal Society of London, Series A 380 (1982), 171-185. MR 83d:83019

[13] C.R. LeBrun, Twistors, Ambitwistors, and Conformal Gravity, in Twistors in mathematics and physics, London Math. Soc. Lecture Note Ser., 156 (1990), Cambridge Univ. Press, Cambridge, 71-86. MR 92a:83042

[14] C.R. LeBrun, Foliated CR Manifolds, Journal of Differential Geometry 22 (1985), 81-96. MR 88e:32028

[15] C.R. LeBrun, Spaces of Complex Null Geodesics in Complex-Riemannian Geometry, Trans. Am. Math. Soc. 278 (1983), 209-231. MR 84e:32023

[16] R. Penrose, The Structure of Space-Time, in Battelle Rencontres (ed. C. DeWitt \& J. Wheeler), New York: Benjamin (1968), 121-235. MR 38:955

[17] R. Penrose, Nonlinear Gravitons and Curved Twistor Theory, General Relativity and Gravitation 7 (1976), 31-52. MR 55:11905

[18] S. Salamon, Topics in four-dimensional Riemannian geometry, in Geometry Seminar "Luigi Bianchi”, Lecture Notes in Mathematics 1022 (1982) Springer-Verlag, 36-124. MR 85i:53002

[19] I.M. Singer, J.A. Thorpe, The curvature of 4-dimensional Einstein spaces, in Global Analysis, in honor of Kodaira, Princeton Math. Series 29 (1969), Princeton Univ. Press, 355-365. MR 41:959

[20] T.Y. Thomas, A projective theory of affinely connected manifolds, Math. Zeit. 25 (1926), 723-733.

[21] J.H.C. Whitehead, Convex regions in the geometry of paths, Quart. J. Math. 3 (1932), 33-42.

Centre de Mathématiques, UmR 7640 CnRs, Ecole Polytechnique, 91128 Palaiseau CEDEx, France

E-mail address: belgun@math.polytechnique.fr

Current address: Mathematisches Institut, Augustusplatz 10/11, 04109 Leipzig, Germany

E-mail address: Florin.Belgun@math.uni-leipzig.de 